\title{
Modular application of an Integration by Fractional Expansion (IBFE) method to multiloop Feynman diagrams
}

\author{
Iván González* \\ Departamento de Física \\ Pontificia Universidad Católica de Chile \\ Santiago, Chile \\ Iván Schmidt ${ }^{\dagger}$ \\ Departamento de Física y Centro de Estudios Subatómicos \\ Universidad Técnica Federico Santa María \\ Valparaiso, Chile
}

\begin{abstract}
We present an alternative technique for evaluating multiloop Feynman diagrams, using the integration by fractional expansion method. Here we consider generic diagrams that contain propagators with radiative corrections which topologically correspond to recursive constructions of bubble type diagrams. The main idea is to reduce these subgraphs, replacing them by their equivalent multiregion expansion. One of the main advantages of this integration technique is that it allows to reduce massive cases with the same degree of difficulty as in the massless case.
\end{abstract}

PACS : 11.25.Db; 12.38.Bx

Keywords : Perturbation theory; Scalar integrals; Multiloop Feynman diagrams; Schwinger parameters; Negative Dimension Integration Method (NDIM), integration by fractional expansion (IBFE).

*e-mail: igonzalez@fis.puc.cl

†e-mail: ivan.schmidt@usm.cl 


\section{Introduction}

The evaluation of multiloop Feynman diagrams is currently one of the most important problems in Quantum Field Theory. Thanks to the development of analytical techniques in high order perturbative calculations, it has become possible to compare precision experimental measurements with the theoretical models that try to explain them. There are several of these techniques, and some of the best known can even be found in textbooks $[1,2]$. One that is not particularly used but which nevertheless has advantages for the evaluation of some complicated Feynman diagrams is called Negative Dimension Integration Method (NDIM). The basic foundations of NDIM were initially suggested in the work of Halliday and Ricotta [3], which using the dimensional regularization prescription $(D=4-2 \epsilon)$ make an analytical continuation of the dimension $D$ into negative values, something which can be done since the Feynman integrals are in fact analytic in arbitrary dimension $D$. In a previous work [4] we proposed that a more appropriate name for this technique should be integration by fractional expansion (IBFE), representing better its mathematical and physical basis.

The purpose of the IBFE technique is to transform the Schwinger integral parametric representation of a specific Feynman diagram into an equivalent mathematical structure, which contains several summations and Kronecker deltas and which we call multiregion expansion (MRE) of the diagram. This name comes from the fact that this particular expansion is made around the values zero and infinity simultaneously, and that once the expression is summed using the Kronecker deltas, these expansions become explicitly separated. In general the number of summations is bigger than the number of deltas that are generated, and in this case there are several different ways of summing using the Kronecker deltas, in fact $C_{\delta}^{\sigma}=\frac{\sigma !}{\delta !(\sigma-\delta) !}$ different forms, where $\delta$ is the number of Kronecker deltas and $\sigma$ the number of sums. Each one of these forms give rises in general to generalized and multivariable hypergeometric functions.

Several authors have used and formalized this method, with a wide variety of applications to mainly one loop $[6,7,8,9,10]$ and two loop $[11,12,13,14,15]$ diagrams. In a previous work we presented a optimization of this technique [4], which allows to evaluate more complex diagrams and even certain families of $L$ loop diagrams. In this work we will show that is possible to improve the efficiency of the IBFE method when it is applied in a modular form to a diagram, that is applying it iteratively to subgraphs or modules that contain one or more contiguous loops of the same diagram. The procedure consists in replacing the Schwinger integral parametric representation of each module by its corresponding MRE, and simultaneously the diagram is reduced topologically into simpler graphs. This reduction allows to get the MRE of the complete diagram, as a product of functions that we have called $n$-loop functions (associated to $n$ loop subgraphs), each of them being an MRE related to one of the modules that are present in the diagram. Since each module can have different masses distributed in different ways in its propagators, there exist several $n$-loop functions depending on the particular configuration of masses in the propagators.

The optimization that is reached when applying the IBFE method in this way to a diagram consists in a reduction of the obtained MRE with respect to the one that is reached when the diagram is considered with all its loops simultaneously. In order to explain the technique in detail, we start with the analysis of the simplest topology that can constitute a subgraph of a Feynman diagram: the one loop module (subgraph), the bubble diagram, which is going to be the basis that will be used to derive 1-loop functions, useful to evaluate a variety of Feynman diagrams.

The remainder of the paper is organized as follows. In Sec. II, we describe how to easily obtain the Schwinger parametric representation associated to a Feynman diagram, whose mathematical structure is the starting point for the application of the IBFE technique. Here we present the basic elements of the integration method, and a more rigorous extension is given in the Appendix. In section III we briefly review the conventional way to deal with this type of diagrams, which is loop by loop. This will provide a direct test for the method proposed here. Later, in Sections IV and V we define and deduce the 1-loop functions of the bubble module, for all the possible mass and momentum configurations, which are a total of eight functions : $G_{k}$ and $\bar{G}_{k}(k=A, B, C, D)$. Finally, in Section VI we develop several applications of the proposed modular procedure, which will be compared with its equivalent MRE obtained from an application of the IBFE technique considering all loops at the same time. 


\section{Mathematical Formalism}

In what follows we will briefly describe the algebraic elements that are needed to understand the technique.

\subsection{Schwinger's parametric representation}

The IBFE integration technique is applied directly to the Schwinger parametric representation of a diagram, and therefore it is important to have a simple algorithm to obtain this representation, which is what we will do in this subsection.

Let us consider a generic topology $G$, which represents a Feynman diagram in a scalar theory, and let us suppose that the graph has: $N$ propagators, each one associated to the masses $\left\{m_{1}, \ldots, m_{N}\right\} ; L$ loops, associated to the independent internal momenta $\left.\left\{q_{1}, \ldots, q_{L}\right\}\right)$; and $E$ independent external momenta $\left\{p_{1}, \ldots, p_{E}\right\}$.

Using the dimensional regularization prescription we can write the momentum space integral expression that represents the diagram in $D=4-2 \epsilon$ dimensions:

$$
G=\int \frac{d^{D} q_{1}}{i \pi^{\frac{D}{2}}} \ldots \frac{d^{D} q_{L}}{i \pi^{\frac{D}{2}}} \frac{1}{\left(B_{1}^{2}-m_{1}^{2}+i 0\right)^{\nu_{1}}} \ldots \frac{1}{\left(B_{N}^{2}-m_{N}^{2}+i 0\right)^{\nu_{N}}} .
$$

In this expression the $B_{j}$ symbol represents the momentum of the $j$ propagator, whose dependence in general is given as a linear combination of external and internal momenta. We also define $\left\{\nu_{1}, \ldots, \nu_{N}\right\}$ as the set of propagator indices or powers, and which are considered to have arbitrary values. After introducing Schwinger's parametrization it is possible to evaluate the momentum integrals as gaussian integrals, and the result of this operation is Schwinger's parametric representation of (1), which in the general case is going to be given by an expression of the form:

$$
G=\frac{(-1)^{-\frac{L D}{2}}}{\prod_{j=1}^{N} \Gamma\left(\nu_{j}\right)} \int_{0}^{\infty} d \vec{x} \frac{\exp \left(\sum_{j=1}^{N} x_{j} m_{j}^{2}\right) \exp \left(-\frac{F}{U}\right)}{U^{\frac{D}{2}}} .
$$

For simplicity we have introduced the notation $d \vec{x}=d x_{1} \ldots d x_{N} \prod_{j=1}^{N} x_{j}^{\nu_{j}-1}$, where $F$ is defined as:

$$
F=\sum_{i, j=1}^{E} C_{i j} p_{i} \cdot p_{j}
$$

The function $U$ and the coefficients $C_{i j}$ are $L$-lineal and $(L+1)$-lineal homogeneous polynomials (Symanzik or Kirchhoff polynomials [16] ) respectively, in the Schwinger parameters. Both $U$ and $C_{i j}$ correspond to determinants related to a matrix that we have called matrix of parameters $\mathbf{M}$ [5]. An important characteristic of the coefficients $C_{i j}$ is that they are symmetric $\left(C_{i j}=C_{j i}\right)$, which is due to the commutativity of the internal product between the independent external momenta associated to the graph $G$. These determinants are defined through the following expressions:

$$
\begin{gathered}
U=\left|\begin{array}{ccc}
M_{11} & \cdots & M_{1 L} \\
\vdots & & \vdots \\
M_{L 1} & \cdots & M_{L L}
\end{array}\right|, \\
C_{i j}=\left|\begin{array}{lllc}
M_{11} & \cdots & M_{1 L} & M_{1(L+j)} \\
\vdots & & \vdots & \vdots \\
M_{L 1} & \cdots & M_{L L} & M_{L(L+j)} \\
M_{(L+i) 1} & \cdots & M_{(L+i) L} & M_{(L+i)(L+j)}
\end{array}\right| .
\end{gathered}
$$


As can be seen, the determinants are associated to submatrices of the matrix of parameters $\mathbf{M}$, which is symmetric and of dimension $(L+E) \times(L+E)$. The actual form of this matrix can be easily obtained when (1) is parameterized and the internal products of all the (internal and external) momenta associated to $G$ are expanded, arriving at a quadratic form. The coefficients of such an expansion correspond to the matrix elements $M_{i j}$. For a better understanding of this process let us define for convenience the momentum:

$$
Q_{j}= \begin{cases}q_{j} & \text { if } \quad L \geq j \geq 1 \\ p_{j-L} & \text { if } \quad(L+E) \geq j>L,\end{cases}
$$

with which one can build the $(L+E)$-vector $\mathbf{Q}=\left[\begin{array}{llll}Q_{1} & Q_{2} & \ldots & Q_{(L+E)}\end{array}\right]^{\mathbf{t}}$. Using this definition the following matrix structure is generated in the integral, after the parametrization application and before the loop momenta integration:

$$
G=\frac{1}{\prod_{j=1}^{N} \Gamma\left(\nu_{j}\right)} \int_{0}^{\infty} d \vec{x} \exp \left(\sum_{j=1}^{N} x_{j} m_{j}^{2}\right) \int \prod_{j=1}^{L}\left(\frac{d^{D} Q_{j}}{i \pi^{D / 2}}\right) \exp \left(-\sum_{i=1}^{L+E} \sum_{j=1}^{L+E} Q_{i} M_{i j} Q_{j}\right),
$$

and from which we can identify the symmetric matrix $\mathbf{M}$.

\subsection{Foundations of the integration method IBFE}

\subsubsection{Notation and fundamental formulae}

This technique can be introduced directly by considering the integral expression for the Gamma function. The idea is to obtain operational rules, which will allow us later on to work with generalized complicated structures of this type of integrals, such as Schwinger's parametric representation of a generic Feynman diagram (2). Let us analyze the following integral structure:

$$
\frac{1}{A^{\beta}}=\frac{1}{\Gamma(\beta)} \int_{0}^{\infty} d x x^{\beta-1} \exp (-A x)
$$

where the quantities $A$ and $\beta$ are arbitrary. Expanding the integrand we get:

$$
\frac{1}{A^{\beta}}=\frac{1}{\Gamma(\beta)} \sum_{n} \phi_{n} A^{n} \int_{0}^{\infty} d x x^{\beta+n-1}
$$

where we have defined the factor:

$$
\phi_{n}=\frac{(-1)^{n}}{\Gamma(n+1)} .
$$

Now the integral evaluation will not be done in the usual way, but we define the following operational relation:

$$
\int_{0}^{\infty} d x x^{\beta+n-1} \equiv \Gamma(\beta) \frac{\Gamma(n+1)}{(-1)^{n}} \delta_{\beta+n, 0}
$$

which makes (9) to be an identity: 


$$
\begin{aligned}
\frac{1}{A^{\beta}} & =\frac{1}{\Gamma(\beta)} \sum_{n} \frac{(-1)^{n}}{\Gamma(n+1)} A^{n} \int_{0}^{\infty} d x x^{\beta+n-1}=\frac{1}{\Gamma(\beta)} \sum_{n} \frac{(-1)^{n}}{\Gamma(n+1)} A^{n}\left(\Gamma(\beta) \frac{\Gamma(n+1)}{(-1)^{n}} \delta_{\beta+n, 0}\right) \\
& =\sum_{n} A^{n} \delta_{\beta+n, 0} \\
& =A^{-\beta} .
\end{aligned}
$$

For convenience we introduce the following notation:

$$
\int d x x^{(\alpha+\beta)-1} \equiv\langle\alpha+\beta\rangle,
$$

where the parenthesis $\langle\cdot\rangle$ has implicit the constraint associated to the Kronecker delta. This identity is the first fundamental formula of the integration method IBFE. With the previously defined notation, equation (9) can be written in the following way:

$$
\frac{1}{A^{\beta}}=\frac{1}{\Gamma(\beta)} \sum_{n} \phi_{n} A^{n}\langle\beta+n\rangle,
$$

which corresponds to the multiregion expansion (MRE) of the factor $A^{-\beta}$.

On the other hand, starting from the application of equation (8) and later equation (13) to an arbitrary multinomial, we find the second fundamental formula that the method uses. This expresses the fact that a multinomial of $\sigma$ terms can be written as a MRE, in such a way that it contains simultaneously all the possible expansions with respect to the ratio of the different terms that are present in the multinomial. This MRE can be written in the following form:

$$
\left(A_{1}+\ldots+A_{\sigma}\right)^{ \pm \nu}=\sum_{n_{1}} \ldots \sum_{n_{\sigma}} \phi_{n_{1}, . ., n_{\sigma}} A_{1}^{n_{1}} \ldots A_{\sigma}^{n_{\sigma}} \frac{\left\langle\mp \nu+n_{1}+\ldots+n_{\sigma}\right\rangle}{\Gamma(\mp \nu)}
$$

where the definition of the factor (10) has been generalized to:

$$
\phi_{n_{1}, \ldots, n_{\sigma}}=\phi_{n_{1}} \ldots \phi_{n_{\sigma}}=(-1)^{n_{1}+\ldots+n_{\sigma}} \frac{1}{\Gamma\left(n_{1}+1\right) \ldots \Gamma\left(n_{\sigma}+1\right)} .
$$

\subsubsection{General form of a diagram MRE and its solutions}

Once the parametric representation of the diagram (2) has been obtained, the following step is finding its MRE, and for this it is necessary to expand the integrand starting with the exponentials if they exist, and then expanding all the multinomials that the procedure is generating according to formula (15). This expansion process stops when finally there is only one term, which is a product of all the Schwinger parameters. At this point all that is left is to replace the integrals according to formula (13) into its equivalent $\langle\cdot\rangle$. The result is the MRE of the Feynman integral $G$ considered in (1). In the case of a general topology characterized by $M$ different mass scales, $N$ propagators, $L$ loops and a minimal quantity $P$ of invariants associated to scalar products of the independent external momenta, then the general form of the MRE of $G$ is given by the following expression:

$$
G=(-1)^{-\frac{L D}{2}} \sum_{n_{1}, . ., n_{\sigma}} \phi_{n_{1}, . ., n_{\sigma}} \prod_{i=1}^{P}\left(Q_{i}^{2}\right)^{n_{i}} \prod_{j=P+1}^{P+M}\left(-m_{j}^{2}\right)^{n_{j}} \prod_{k=1}^{N} \frac{\left\langle\nu_{k}+\alpha_{k}\right\rangle}{\Gamma\left(\nu_{k}\right)} \prod_{l=1}^{K} \frac{\left\langle\beta_{l}+\gamma_{l}\right\rangle}{\Gamma\left(\beta_{l}\right)}
$$

where we can identify the following quantities:

- $\sigma \Longrightarrow$ Multiplicity or number of sums that are present in the MRE of diagram $G$.

- $Q_{j}^{2} \Longrightarrow$ A kinematical invariant, which is a quadratic form of the independent external momenta. 
- $\alpha_{j}, \beta_{j}, \gamma_{j} \Longrightarrow$ Linear combinations of the indexes $\left\{n_{1}, . ., n_{\sigma}\right\}$, except for $\beta_{1}$, which is the only one that has dependence on the dimension $D$ :

$$
\beta_{1}=\frac{D}{2}+n_{1}+\ldots+n_{P}
$$

The coefficients of the indices of the sum $\left\{n_{i}\right\}$ in the linear combinations $\alpha_{j}$ and $\gamma_{j}$ are $(+1)$, and in the case of $\beta_{j}$ the indices have coefficients $(-1)$, except for $\beta_{1}$.

- $N \Longrightarrow$ Number of propagators or equivalently number of parametric integrations, which the method transforms into $N$ Kronecker deltas.

- $K \Longrightarrow$ Total number of MREs performed over the integrand of the parametric representation, which in turn generates $K$ constraints or equivalently $K$ Kronecker deltas. This only refers to multinomial expansions that are present in the parametric integral.

In order to find the solutions it is necessary to evaluate the sums that are present in (17), using for this purpose the existing constraints between the sum indices, represented by the $\delta=(N+K)$ Kronecker deltas. There are several ways to do this evaluation, and in fact the number of different ways to evaluate the MRE of $G$ using the Kronecker deltas is given by the combinatorial formula:

$$
C_{\delta}^{\sigma}=\frac{\sigma !}{\delta !(\sigma-\delta) !} .
$$

Each of these forms of summing will generate as a result a term in the solution of $G$, which corresponds to a generalized hypergeometric function, and whose multiplicity is given by:

$$
\mu=(\sigma-\delta) .
$$

In general it is not always possible to use the $\delta$ Kronecker deltas to evaluate a similar number of sums, since this will depend on the combination of sum indices for which the sum is going to be done. If this happens, these cases simply do not generate contributions to the solution.

In simple terms, the idea of the method is to generate finally an expansion that represents the diagram $G$, the MRE of $G$, characterized by a multiplicity $\sigma$ in combination with $\delta$ Kronecker deltas. From this it is possible to get the solution in terms of generalized hypergeometric functions, series of multiplicity $\mu$ whose arguments usually correspond to ratios between two characteristic energy scales of the diagram, or in the more general case include sums of unitary argument.

\section{Conventional reduction of diagrams with massless bubble inser- tions}

\subsection{Graphical representation of the reduction}

Many diagrams contain bubble type insertions in the propagators, which in the massless case can be directly reduced to a propagator and a factor which is a ratio of Gamma functions. This result is straightforward and is very useful for an iterative or loop by loop reduction of topologies or subtopologies built in terms of bubble type insertions. The fundamental formula for insertions or bubble modules can be deduced directly from the solution of the momentum integral for this diagram:

$$
G=\int \frac{d^{D} q}{i \pi^{D / 2}} \frac{1}{\left(q^{2}\right)^{a_{1}}\left((q+p)^{2}\right)^{a_{2}}}
$$

where the indices $a_{1}$ and $a_{2}$ are arbitrary. The explicit solution of this integral is:

$$
G=g\left(a_{1}, a_{2}\right) \frac{1}{\left(p^{2}\right)^{a_{1}+a_{2}-\frac{D}{2}}},
$$


where the factor $g\left(a_{1}, a_{2}\right)$ is given by:

$$
g\left(a_{1}, a_{2}\right)=(-1)^{-\frac{D}{2}} \frac{\Gamma\left(a_{1}+a_{2}-\frac{D}{2}\right) \Gamma\left(\frac{D}{2}-a_{1}\right) \Gamma\left(\frac{D}{2}-a_{2}\right)}{\Gamma\left(a_{1}\right) \Gamma\left(a_{2}\right) \Gamma\left(D-a_{1}-a_{2}\right)} .
$$

Usually equation (22) is represented pictorially in the following way:

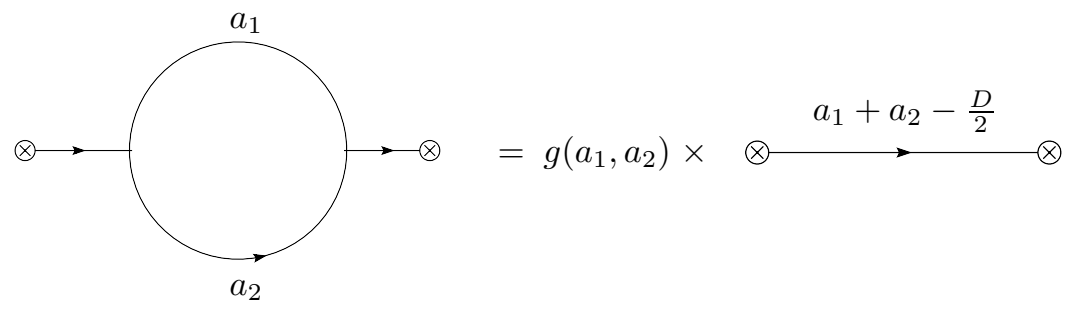

Another graphical formula, which is useful when two or more scalar propagators are in series, is:

$$
\otimes \longrightarrow a_{1}^{a_{1}} \otimes \stackrel{a_{2}}{\longrightarrow} \otimes=\stackrel{\left(a_{1}+a_{2}\right)}{\longrightarrow} \otimes
$$

This last formula is also valid for a massive theory in the case of two equal masses. This two pictorial expressions are enough in order to find the solution of this family of diagrams. Finally the operational problem consists in literally reducing series and parallel propagators, and the formulae (24) and (25) represent this operation.

\subsection{A simple application}

In order to show the usefulness of the previous pictorial formulae (24) and (25), let us consider the following radiative correction to a propagator through which it flows a momentum $p$ :

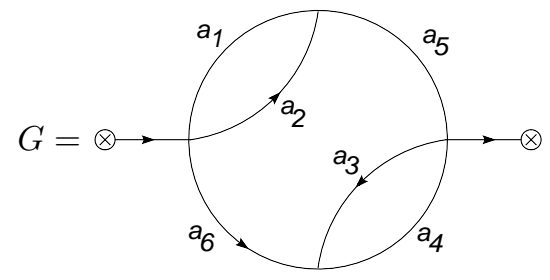

Then, using equation (24) we can reduce the two bubble type subgraphs that appear in the diagram, and we get:

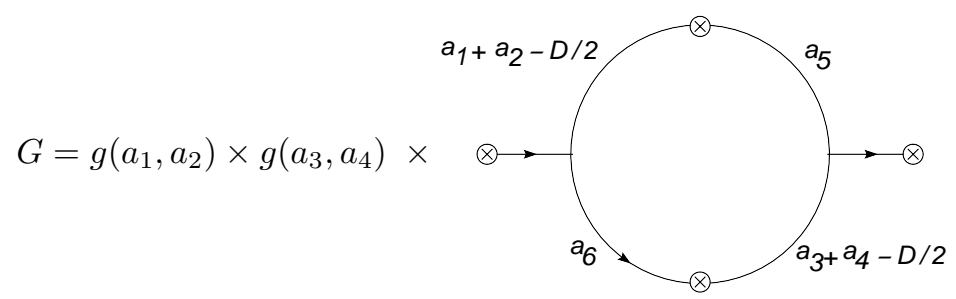

The resulting loop is easily evaluated after using equation (25), and this finally allows us to get the solution of this diagram:

$$
G=g\left(a_{1}, a_{2}\right) \times g\left(a_{3}, a_{4}\right) \times g\left(a_{1}+a_{2}+a_{5}-\frac{D}{2}, a_{3}+a_{4}+a_{6}-\frac{D}{2}\right) \times \otimes \stackrel{a_{1}+\ldots+a_{6}-\frac{3 D}{2}}{\longrightarrow} \otimes,
$$

which is equivalent to the following expression: 


$$
G=g\left(a_{1}, a_{2}\right) \times g\left(a_{3}, a_{4}\right) \times g\left(a_{125}-\frac{D}{2}, a_{346}-\frac{D}{2}\right) \frac{1}{\left(p^{2}\right)^{a_{1}+\ldots+a_{6}-\frac{3 D}{2}}}
$$

To simplify the notation we have defined for the sum of indices the following symbology:

$$
a_{i j k \ldots}=a_{i}+a_{j}+a_{k}+\ldots
$$

Using then formula (23) we finally obtain the explicit solution for the diagram:

$$
\begin{aligned}
G= & (-1)^{-\frac{3 D}{2}} \frac{\Gamma\left(a_{12}-\frac{D}{2}\right) \Gamma\left(\frac{D}{2}-a_{1}\right) \Gamma\left(\frac{D}{2}-a_{2}\right)}{\Gamma\left(a_{1}\right) \Gamma\left(a_{2}\right) \Gamma\left(D-a_{12}\right)} \frac{\Gamma\left(a_{34}-\frac{D}{4}\right) \Gamma\left(\frac{D}{4}-a_{3}\right) \Gamma\left(\frac{D}{4}-a_{4}\right)}{\Gamma\left(a_{3}\right) \Gamma\left(a_{4}\right) \Gamma\left(D-a_{34}\right)} \\
& \frac{\Gamma\left(a_{123456}-\frac{3 D}{2}\right) \Gamma\left(D-a_{125}\right) \Gamma\left(D-a_{346}\right)}{\Gamma\left(a_{125}-\frac{D}{2}\right) \Gamma\left(a_{346}-\frac{D}{2}\right) \Gamma\left(2 D-a_{123456}\right)} \frac{1}{\left(p^{2}\right)^{a_{1}+\ldots+a_{6}-\frac{3 D}{2}}} .
\end{aligned}
$$

\section{IBFE and modular reduction of diagrams}

\subsection{The bubble module}

The application of IBFE to the bubble module will allow us to find the MREs that generate the 1-loop functions which identify the different masses and momenta configurations that can be associated to this diagram. We start our analysis by considering this diagram to be associated to the kinematical variable $p^{2}$, and then from this we can define the following associated 1-loop functions: $G_{k}$ and $\bar{G}_{k}(k=A, B, C, D)$, according to the value of this variable and the corresponding mass configuration for this module:

- Case $p^{2} \neq 0$

$G_{A} \Longrightarrow$ Massless propagators.

$G_{B} \Longrightarrow$ One massive propagator.

$G_{C} \Longrightarrow$ Equal mass propagators.

$G_{D} \Longrightarrow$ Different mass propagators.

- Case $p^{2}=0$ : This could be obtained from the previous functions, just by taking the kinematical variable to zero. Nevertheless, we will take a different approach, which uses the series multiregion representation obtained directly from the parametric representation of a vacuum bubble. The reason is simple, in general the mathematical expression for the MRE is simpler than the corresponding expression obtained by taking $p^{2}=0$ in the functions $G_{k}$. These functions are useful when diagrams associated with vacuum fluctuations are evaluated. The 1-loop functions that we will define for this case are analogous to the previous ones:

$$
\begin{aligned}
& \bar{G}_{A} \Longrightarrow \text { Massless propagators. } \\
& \bar{G}_{B} \Longrightarrow \text { One massive propagator. } \\
& \bar{G}_{C} \Longrightarrow \text { Equal mass propagators. } \\
& \bar{G}_{D} \Longrightarrow \text { Different mass propagators. }
\end{aligned}
$$

For our analysis let us start by writing the general integral representation of this diagram in momentum space:

$$
G=\int \frac{d^{D} q}{i \pi^{D / 2}} \frac{1}{\left(q^{2}-M_{j}^{2}\right)^{a_{j}}\left((p-q)^{2}-M_{k}^{2}\right)^{a_{k}}} .
$$


We shall consider general cases, so the powers $a_{j}$ and $a_{k}$ are arbitrary. We then obtain Schwinger's parametric representation:

$$
G=\frac{(-1)^{-\frac{D}{2}}}{\Gamma\left(a_{j}\right) \Gamma\left(a_{k}\right)} \int_{0}^{\infty} d \vec{x} \frac{\exp \left(x_{j} M_{j}^{2}\right) \exp \left(x_{k} M_{k}^{2}\right) \exp \left(-\frac{x_{j} x_{k}}{x_{j}+x_{k}} p^{2}\right)}{\left(x_{j}+x_{k}\right)^{\frac{D}{2}}} .
$$

Depending on the specific values of the masses $\left\{M_{j}, M_{k}\right\}$ and of $\left\{p^{2}\right\}$, we can consider eight possible 1-loop functions, which will be discussed in what follows.

\subsection{1-loop functions for $p^{2} \neq 0$}

There are four loop functions that can be defined in this case $\left(G_{A}, G_{B}, G_{C}\right.$ and $\left.G_{D}\right)$, of which only the last two are independent. The loop function $G_{A}$ is a particular case of $G_{C}$ or $G_{D}$, and in the same way $G_{B}$ turns out to be a particular case of $G_{D}$. This will be shown now.

\subsubsection{1-loop function $G_{D} \Longrightarrow M_{j} \neq M_{k} \neq 0$}

Let us start with the most general case, with propagators with different masses. Then the corresponding parametric integral is given through equation (33), and the first step is to fractionally expand the exponential that contains the invariant $p^{2}$, which gives:

$$
G=\frac{(-1)^{-\frac{D}{2}}}{\Gamma\left(a_{j}\right) \Gamma\left(a_{k}\right)} \sum_{n} \phi_{n}\left(p^{2}\right)^{n} \int_{0}^{\infty} d \vec{x} \exp \left(x_{j} M_{j}^{2}\right) \exp \left(x_{k} M_{k}^{2}\right) \frac{x_{j}^{n} x_{k}^{n}}{\left(x_{j}+x_{k}\right)^{\frac{D}{2}+n}}
$$

or equivalently:

$$
G=\sum_{n} G_{D}\left(a_{j}, a_{k} ; n ; M_{j}^{2}, M_{k}^{2}\right)\left(p^{2}\right)^{n}=\sum_{n} G_{D}\left(a_{j}, a_{k} ; n ; M_{j}^{2}, M_{k}^{2}\right) \frac{1}{\left(p^{2}\right)^{-n}},
$$

where the 1-loop function has been defined as:

$$
G_{D}\left(a_{j}, a_{k} ; n ; M_{j}^{2}, M_{k}^{2}\right)=\frac{(-1)^{-\frac{D}{2}}}{\Gamma\left(a_{j}\right) \Gamma\left(a_{k}\right)} \phi_{n} \int_{0}^{\infty} d \vec{x} \exp \left(x_{j} M_{j}^{2}\right) \exp \left(x_{k} M_{k}^{2}\right) \frac{x_{j}^{n} x_{k}^{n}}{\left(x_{j}+x_{k}\right)^{\frac{D}{2}+n}} .
$$

Remember that the factor $\phi_{n}$ is given by $\phi_{n}=\frac{(-1)^{n}}{\Gamma(n+1)}$. The following step is finding the MRE of the loop function $G_{D}\left(a_{j}, a_{k} ; n ; M_{j}^{2}, M_{k}^{2}\right)$, and for this purpose we expand the mass exponentials in (36) and then the binomial in the denominator of the same formula, which gives:

$$
\frac{1}{\left(x_{j}+x_{k}\right)^{\frac{D}{2}+n}}=\sum_{l_{j}, l_{k}} \phi_{l_{j}, l_{k}} x_{j}^{l_{j}} x_{k}^{l_{k}} \frac{\left\langle\frac{D}{2}+n+l_{j}+l_{k}\right\rangle}{\Gamma\left(\frac{D}{2}+n\right)},
$$

Performing the necessary algebra, the MRE for $G_{D}\left(a_{j}, a_{k} ; n ; M_{j}^{2}, M_{k}^{2}\right)$ is finally given by:

$$
G_{D}\left(a_{j}, a_{k} ; n ; M_{j}^{2}, M_{k}^{2}\right)=\frac{(-1)^{-\frac{D}{2}}}{\Gamma\left(a_{j}\right) \Gamma\left(a_{k}\right)} \sum_{\substack{m_{j}, m_{k} \\ l_{j}, l_{k}}} \phi_{n, m_{j}, m_{k}, l_{j}, l_{k}}\left(-M_{j}^{2}\right)^{m_{j}}\left(-M_{k}^{2}\right)^{m_{k}} \frac{\Delta_{1} \Delta_{2} \Delta_{3}}{\Gamma\left(\frac{D}{2}+n\right)}
$$

with the constraints being defined by the identities:

$$
\left\{\begin{array}{l}
\Delta_{1}=\left\langle\frac{D}{2}+n+l_{j}+l_{k}\right\rangle \\
\Delta_{2}=\left\langle a_{j}+n+m_{j}+l_{j}\right\rangle \\
\Delta_{3}=\left\langle a_{k}+n+m_{k}+l_{k}\right\rangle
\end{array}\right.
$$


The process of replacing the graph in terms of this equivalent 1-loop function (see equation (35) ) can be written symbolically through the following graphical formulation:

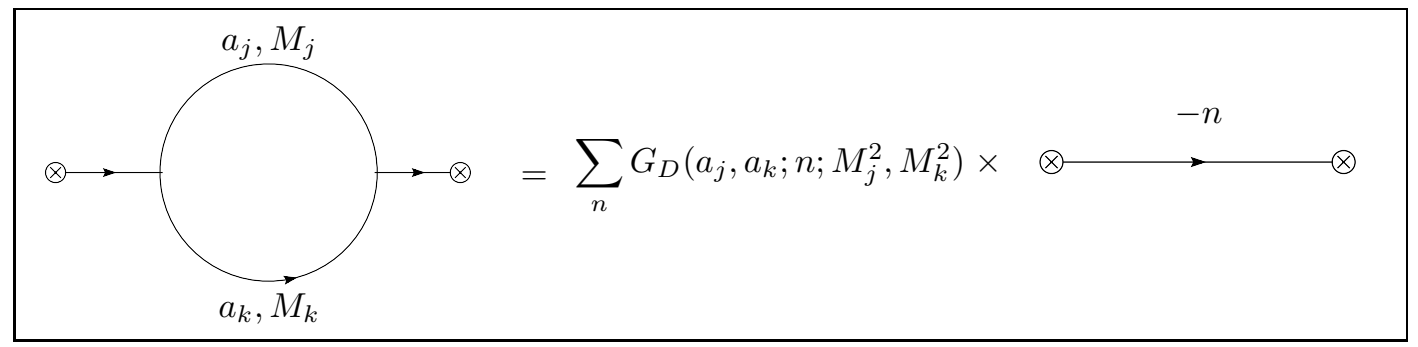

Derivation of $G_{A}$ from $G_{D}$ (Case $\left.M_{j}=M_{k}=0\right)$ : The loop function $G_{A}$ turns out to be a particular case of the 1-loop function $G_{D}$. The function $G_{A}$ can be defined as:

$$
G_{A}\left(a_{j}, a_{k} ; n\right)=G_{D}\left(a_{j}, a_{k} ; n ; 0,0\right),
$$

and whose equivalent MRE corresponds to the expression:

$$
G_{A}\left(a_{j}, a_{k} ; n\right)=\frac{(-1)^{-\frac{D}{2}}}{\Gamma\left(a_{j}\right) \Gamma\left(a_{k}\right)} \sum_{l_{j}, l_{k}} \phi_{n, l_{j}, l_{k}} \frac{\Delta_{1} \Delta_{2} \Delta_{3}}{\Gamma\left(\frac{D}{2}+n\right)}
$$

where the masses were put to zero in (38) and the sums associated to the indices $\left\{m_{j}, m_{k}\right\}$ were eliminated, taking later on these indices to zero. The constraints are now given by the identities:

$$
\left\{\begin{array}{l}
\Delta_{1}=\left\langle\frac{D}{2}+n+l_{j}+l_{k}\right\rangle \\
\Delta_{2}=\left\langle a_{j}+n+l_{j}\right\rangle \\
\Delta_{3}=\left\langle a_{k}+n+l_{k}\right\rangle
\end{array}\right.
$$

As before it is possible to represent the fractional expansion of the graph symbolically through the following graphical equation:

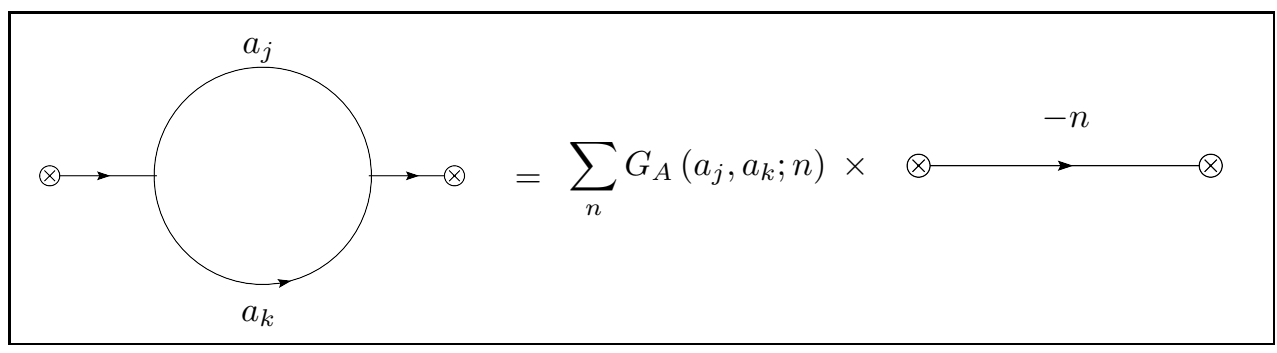

Derivation of $G_{B}$ (Case $\left.M_{j} \neq 0, M_{k}=0\right)$ : The loop function $G_{B}$ can be written in terms of the loop function $G_{D}$ as:

$$
G_{B}\left(a_{j}, a_{k} ; n ; M_{j}^{2}\right)=G_{D}\left(a_{j}, a_{k} ; n ; M_{j}^{2}, 0\right),
$$

the corresponding MRE is obtained just as for $G_{A}$ :

$$
G_{B}\left(a_{j}, a_{k} ; n ; M_{j}^{2}\right)=\frac{(-1)^{-\frac{D}{2}}}{\Gamma\left(a_{j}\right) \Gamma\left(a_{k}\right)} \sum_{m_{j}, l_{j}, l_{k}} \phi_{n, m_{j}, l_{j}, l_{k}}\left(-M_{j}^{2}\right)^{m_{j}} \frac{\Delta_{1} \Delta_{2} \Delta_{3}}{\Gamma\left(\frac{D}{2}+n\right)}
$$

The constraints now are given by: 


$$
\left\{\begin{array}{l}
\Delta_{1}=\left\langle\frac{D}{2}+n+l_{j}+l_{k}\right\rangle \\
\Delta_{2}=\left\langle a_{j}+n+m_{j}+l_{j}\right\rangle \\
\Delta_{3}=\left\langle a_{k}+n+l_{k}\right\rangle
\end{array}\right.
$$

The graphical equation which represents the loop reduction is now:

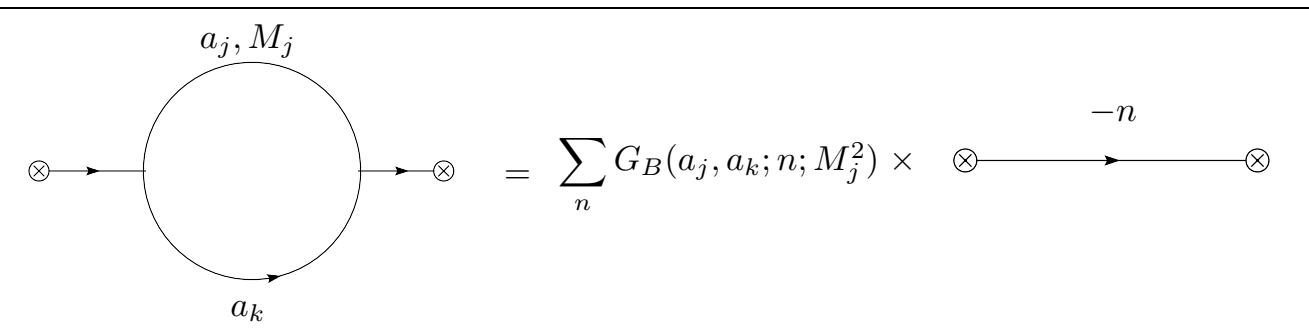

\subsubsection{1-loop function $G_{C} \Longrightarrow M_{j}=M_{k}=M$}

The loop function $G_{C}$ does not correspond to a particularization of $G_{D}$. To see this we start with the parametric representation already factorized:

$$
G=\frac{(-1)^{-\frac{D}{2}}}{\Gamma\left(a_{j}\right) \Gamma\left(a_{k}\right)} \int_{0}^{\infty} d \vec{x} \frac{\exp \left(U M^{2}\right) \exp \left(-\frac{x_{j} x_{k}}{U} p^{2}\right)}{U^{\frac{D}{2}}},
$$

with $U=\left(x_{j}+x_{k}\right)$. The reason that this loop function cannot be derived from $G_{D}$ is related to the denominator structure, where the factorization of the polynomial $U$ produces an MRE of (49) which is more reduced than when we take $M_{j}=M_{k}$ in $G_{D}$.

In equation (49) we expand the exponential which contains the momentum, and obtain the following series:

$$
G=\sum_{n} G_{C}\left(a_{j}, a_{k} ; n ; M^{2}\right) \frac{1}{\left(p^{2}\right)^{-n}}
$$

where we have defined the 1-loop function $G_{C}\left(a_{j}, a_{k} ; n ; M^{2}\right)$ :

$$
G_{C}\left(a_{j}, a_{k} ; n ; M^{2}\right)=\frac{(-1)^{-\frac{D}{2}}}{\Gamma\left(a_{j}\right) \Gamma\left(a_{k}\right)} \phi_{n} \int_{0}^{\infty} d \vec{x} \exp \left(\left(x_{j}+x_{k}\right) M^{2}\right) \frac{x_{j}^{n} x_{k}^{n}}{\left(x_{j}+x_{k}\right)^{\frac{D}{2}+n}},
$$

and expanding the exponential which contains the mass term we have the series:

$$
G_{C}\left(a_{j}, a_{k} ; n ; M^{2}\right)=\frac{(-1)^{-\frac{D}{2}}}{\Gamma\left(a_{j}\right) \Gamma\left(a_{k}\right)} \phi_{n} \sum_{l_{i}} \phi_{l_{i}}\left(-M^{2}\right)^{l_{i}} \int_{0}^{\infty} d \vec{x} \frac{x_{j}^{n} x_{k}^{n}}{\left(x_{j}+x_{k}\right)^{\frac{D}{2}+n-l_{i}}} .
$$

Now we shall find the MRE for the binomial in the integrand denominator:

$$
\frac{1}{\left(x_{j}+x_{k}\right)^{\frac{D}{2}+n-l_{i}}}=\sum_{l_{j}, l_{k}} \phi_{l_{j}, l_{k}} x_{j}^{l_{j}} x_{k}^{l_{k}} \frac{\left\langle\frac{D}{2}+n-l_{i}+l_{j}+l_{k}\right\rangle}{\Gamma\left(\frac{D}{2}+n-l_{i}\right)}
$$

which replaced in (52) and after some algebra allows finally to get the required MRE:

$$
G_{C}\left(a_{j}, a_{k} ; n ; M^{2}\right)=\frac{(-1)^{-\frac{D}{2}}}{\Gamma\left(a_{j}\right) \Gamma\left(a_{k}\right)} \sum_{l_{i}, l_{j}, l_{k}} \phi_{n, l_{i}, l_{j}, l_{k}}\left(-M_{j}^{2}\right)^{l_{i}} \frac{\Delta_{1} \Delta_{2} \Delta_{3}}{\Gamma\left(\frac{D}{2}+n-l_{i}\right)}
$$

where the constraints $\left\{\Delta_{k}\right\}$ are given by: 


$$
\left\{\begin{aligned}
\Delta_{1} & =\left\langle\frac{D}{2}+n-l_{i}+l_{j}+l_{k}\right\rangle, \\
\Delta_{2} & =\left\langle a_{j}+n+l_{j}\right\rangle, \\
\Delta_{3} & =\left\langle a_{k}+n+l_{k}\right\rangle .
\end{aligned}\right.
$$

Then the loop reduction in this case is represented graphically by the following pictorial equation:

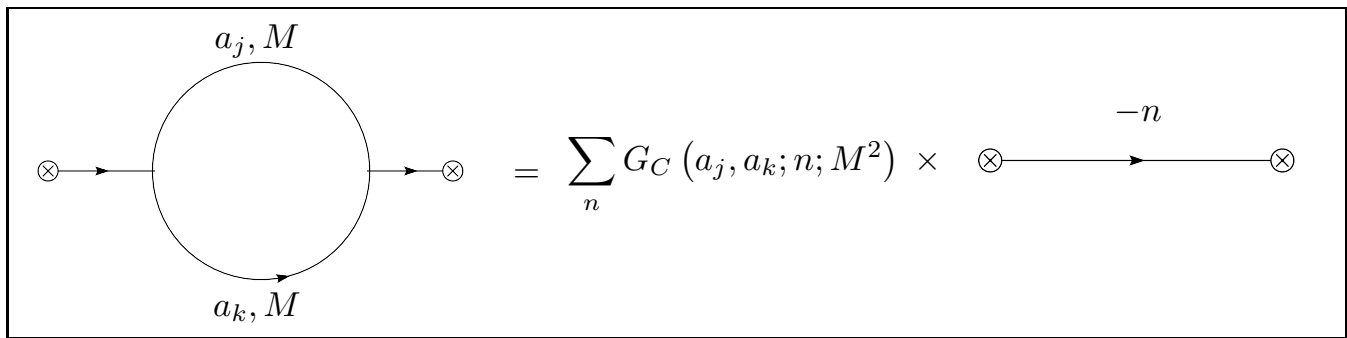

Notice that the 1-loop function $G_{A}$ is also a particular case of $G_{C}$ :

$$
G_{A}\left(a_{j}, a_{k} ; n\right)=G_{C}\left(a_{j}, a_{k} ; n ; 0\right) .
$$

In all the previous representations or reductions of the bubble diagram one can see the similitude with the conventional equation (24). Nevertheless, the differences are important since in the integration by fractional expansion technique (IBFE) massive graphs can be reduced with the same degree of difficulty as in the massless cases.

\section{MREs for one loop vacuum diagrams}

The following 1-loop functions are useful when we wish to evaluate certain topologies associated with vacuum fluctuations. These functions will be identified as $\bar{G}_{K}(K=A, B, C, D)$, in analogy with the ones obtained in the previous section for $p^{2} \neq 0$. The corresponding MREs of these new cases will be obtained directly from their momentum integrals, which corresponds to a bubble without external lines. The resulting MRE for each 1-loop function is in fact simpler than the one obtained by making $p^{2}=0$ in the respective loop function $G_{k}$, that is, it has a more reduced composition of sums and Kronecker deltas.

\subsection{MRE of a bubble with one propagator}

\subsubsection{Massless propagator}

This case does not correspond to any of the bubble diagram formulations previously obtained because it is a bubble with just one propagator. The momentum integral is:

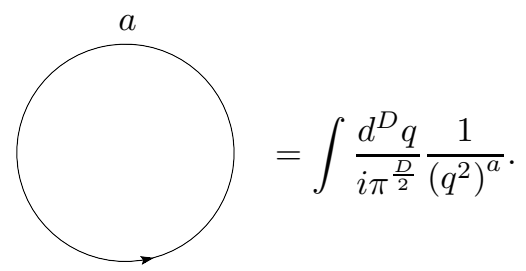

The representation in terms of MRE is for this case easy to find, using:

$$
\begin{aligned}
\int \frac{d^{D} q}{i \pi^{\frac{D}{2}}} \frac{1}{\left(q^{2}\right)^{a}} & =\frac{1}{\Gamma(\nu)} \int_{0}^{\infty} d x x^{a-1} \int \frac{d^{D} q}{i \pi^{\frac{D}{2}}} \exp \left(-x q^{2}\right) \\
& =(-1)^{-\frac{D}{2}} \frac{\left\langle a-\frac{D}{2}\right\rangle}{\Gamma(a)}
\end{aligned}
$$


where we have used the Minskowski space integral identity:

$$
\int \frac{d^{D} q}{i \pi^{\frac{D}{2}}} \exp \left(-\beta q^{2}\right)=\frac{(-1)^{-\frac{D}{2}}}{\beta^{\frac{D}{2}}} .
$$

Finally the required MRE can be written in terms of a pictorial equation as:

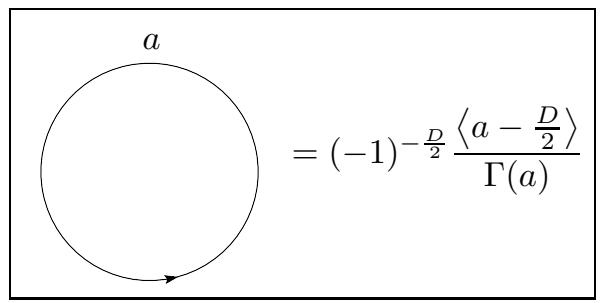

One of the properties of Feynman integrals is that they are invariant under momenta scaling, which allows to show that the integral in equation (58) vanishes, nevertheless, an MRE has been obtained for this diagram. The explanation is quite simple. In fact, first we need to know whether the module constitutes by itself a diagram, or it is s product of a reduction of a more complex diagram. For the last option the MRE does not vanish, but has only validity within the integration method that is used. This allows to formulate a generalization associated to what has been said previously in terms of a theorem:

Any MRE obtained from a Feynman diagram considering all its loops, with a number of constraints (Kronecker deltas)

bigger than the number of summations, vanishes identically.

Let us consider a simple example, a two loop vacuum fluctuation in a massless theory, whose conventional evaluation is rather obvious, but which nevertheless can be an illustrative example of the theorem:

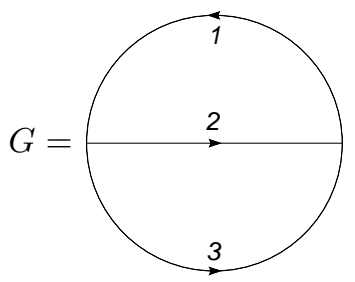

The parametric representation is given by:

$$
G=\frac{(-1)^{-D}}{\Gamma\left(a_{1}\right) \Gamma\left(a_{2}\right) \Gamma\left(a_{3}\right)} \int_{0}^{\infty} d \vec{x} \frac{1}{\left(x_{1} x_{2}+x_{1} x_{3}+x_{2} x_{3}\right)^{\frac{D}{2}}}
$$

Expanding the integrand denominator:

$$
\frac{1}{\left(x_{1} x_{2}+x_{1} x_{3}+x_{2} x_{3}\right)^{\frac{D}{2}}}=\sum_{n_{1}, n_{2}, n_{3}} \phi_{n_{1}, n_{2}, n_{3}} x_{1}^{n_{1}+n_{2}} x_{2}^{n_{1}+n_{3}} x_{3}^{n_{2}+n_{3}} \frac{\left\langle\frac{D}{2}+n_{1}+n_{2}+n_{3}\right\rangle}{\Gamma\left(\frac{D}{2}\right)},
$$

and replacing the integral signs by their respective constraints we obtain the following associated MRE:

$$
G=\frac{(-1)^{-D}}{\Gamma\left(a_{1}\right) \Gamma\left(a_{2}\right) \Gamma\left(a_{3}\right)} \sum_{n_{1}, n_{2}, n_{3}} \phi_{n_{1}, n_{2}, n_{3}} \frac{\left\langle\frac{D}{2}+n_{1}+n_{2}+n_{3}\right\rangle\left\langle a_{1}+n_{1}+n_{2}\right\rangle\left\langle a_{2}+n_{1}+n_{3}\right\rangle\left\langle a_{3}+n_{2}+n_{3}\right\rangle}{\Gamma\left(\frac{D}{2}\right)}
$$


The number of constraints (Kronecker deltas) is bigger than the number of summations, and therefore the previously mentioned theorem says that it should vanish:

$$
G \equiv 0 .
$$

\subsubsection{Massive propagator}

The next degree of difficulty comes from the addition of a mass to the propagator, for which we will use two different ways of finding the corresponding MRE. In the first one the usual formalism will be employed, which consists in parametrizing and then replacing the resulting integral for its corresponding MRE. The second relies in expressing the propagator in terms of its own MRE, and then parametrize the loop integral which now does not contain any mass term.

Alternative I : Direct parametrization of the integral We want to find the MRE of the following Feynman integral:

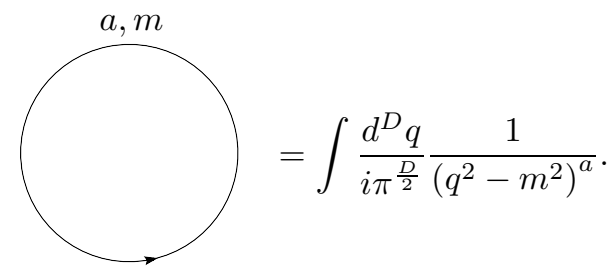

Performing the corresponding algebra allows us to obtain the required MRE:

$$
\begin{aligned}
\int \frac{d^{D} q}{i \pi^{\frac{D}{2}}} \frac{1}{\left(q^{2}-m^{2}\right)^{a}} & =\frac{1}{\Gamma(a)} \int_{0}^{\infty} d x x^{a-1} \exp \left(x m^{2}\right) \int \frac{d^{D} q}{i \pi^{\frac{D}{2}}} \exp \left(-x q^{2}\right) \\
& =\frac{(-1)^{-\frac{D}{2}}}{\Gamma(a)} \sum_{n} \phi_{n}\left(-m^{2}\right)^{n}\left\langle a-\frac{D}{2}+n\right\rangle .
\end{aligned}
$$

Pictorially this is summarized in the following way:

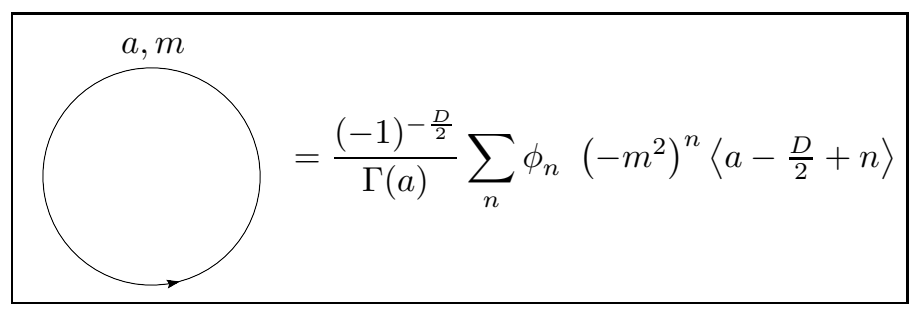

Alternative II : Using the MRE of the massive propagator An alternative form for finding the MRE of this module can be implemented using the MRE of the denominator in the loop integral, which extracts the mass out of the integral and leaves the equivalent of a vacuum massless bubble. Meanwhile, the corresponding MRE of a massive propagator can be easily found using the fundamental equation (15), that is:

$$
\frac{1}{\left(q^{2}-m^{2}\right)^{a}}=\sum_{n_{1}, n_{2}} \phi_{n_{1}, n_{2}}\left(-m^{2}\right)^{n_{1}}\left(q^{2}\right)^{n_{2}} \frac{\left\langle a+n_{1}+n_{2}\right\rangle}{\Gamma(a)} .
$$

We can now rewrite the following equation for a massive bubble: 


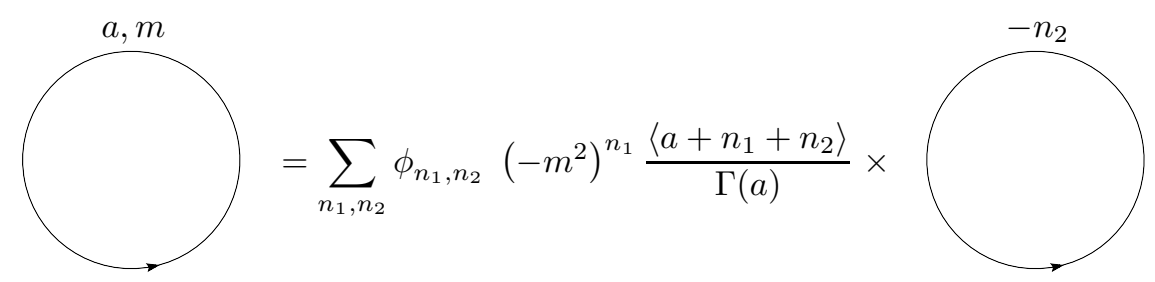

where the massless bubble can be evaluated using equation (61), and then we finally obtain the MRE for this case:

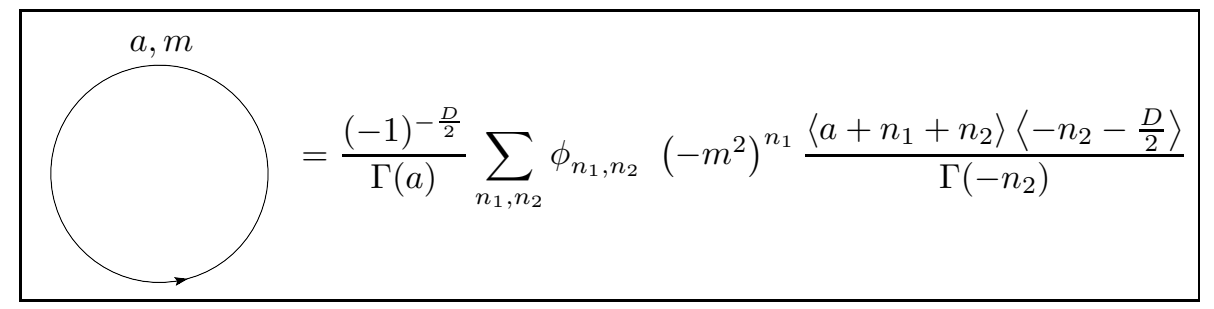

Although (69) and (72) are equivalent, it is clear that (69) is a more compact result, which makes actual evaluation easier.

\subsection{MRE of a bubble with two propagators}

\subsubsection{Trivial cases : $\bar{G}_{A}, \bar{G}_{C}$}

There are two cases of vacuum bubbles with two propagators which are reducible to one propagator. This happens when the two propagators which form the bubble have equal mass parameters, in which case it is possible to use the pictorial equation (25) in order to simplify the graph.

The first case corresponds to a composition of two massless propagators, which results in an expression for the 1-loop function $\bar{G}_{A}$ and which is reduced as follows:

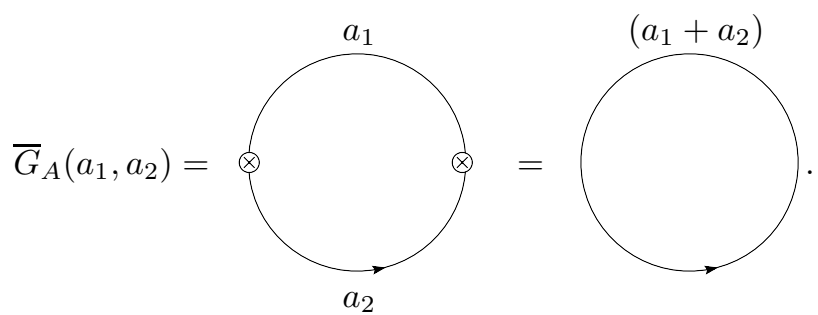

With the help of equation (61) one then obtains the following expression for $\bar{G}_{A}$ :

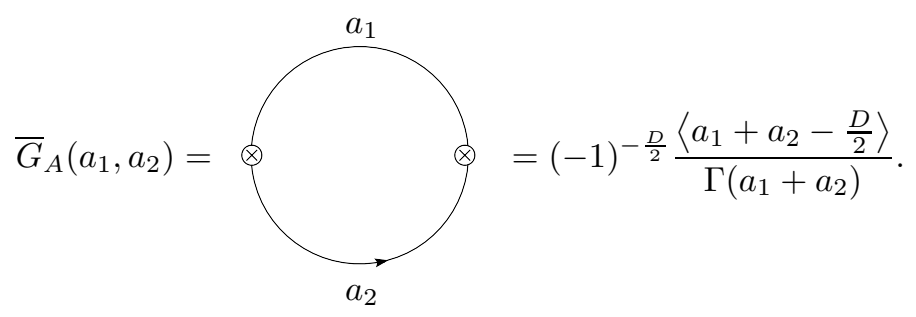

Analogously, for the case of two propagators with the same arbitrary mass $m$, the resulting 1-loop function describes $\bar{G}_{C}$ : 


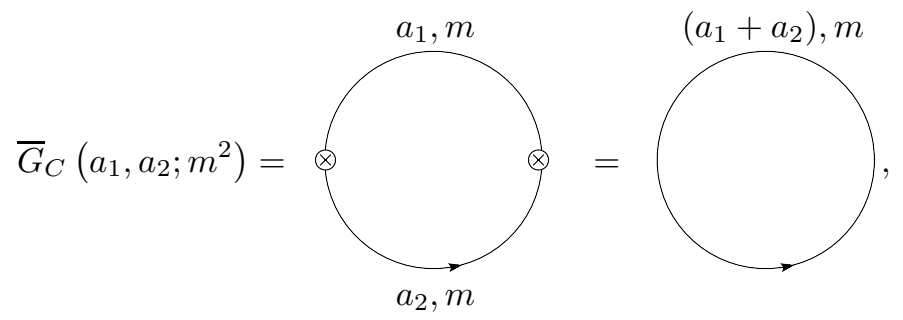

and then using (69) we get the MRE for this module:

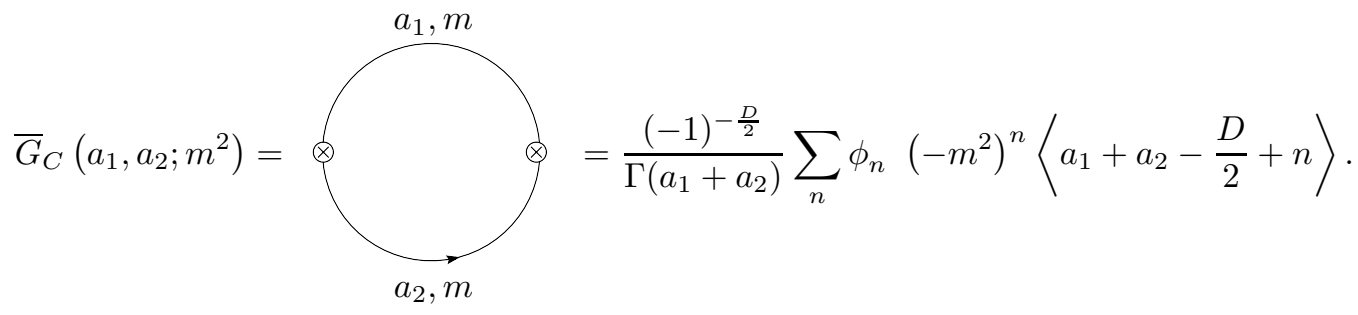

\subsubsection{One massive propagator : $\bar{G}_{B}$}

This case defines the loop function $\bar{G}_{B}$ and just as was done before we will consider two different possible forms to represent the MRE. This module is given by the integral representation:

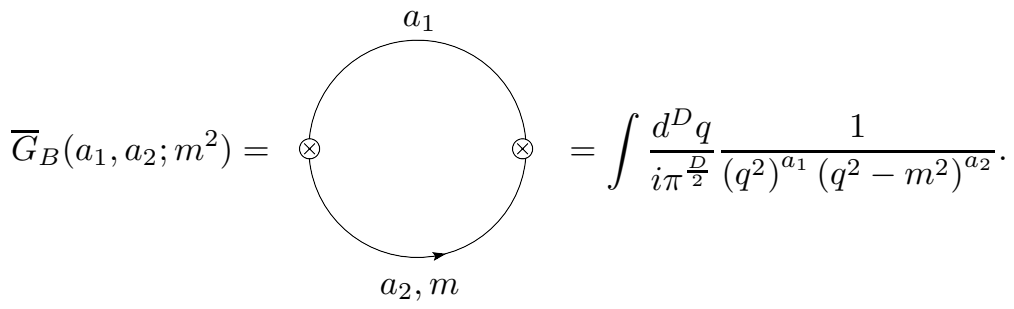

Alternative I : Direct parametrization of the integral The parametrization and subsequent evaluation of the loop integral gives us:

$$
\begin{aligned}
\int \frac{d^{D} q}{i \pi^{\frac{D}{2}}} \frac{1}{\left(q^{2}\right)^{a_{1}}\left(q^{2}-m^{2}\right)^{a_{2}}} & =\frac{1}{\Gamma\left(a_{1}\right) \Gamma\left(a_{2}\right)} \int_{0}^{\infty} d x d y x^{a_{1}-1} y^{a_{2}-1} \exp \left(y m^{2}\right) \int \frac{d^{D} q}{i \pi^{\frac{D}{2}}} \exp \left[-(x+y) q^{2}\right] \\
& =\frac{(-1)^{-\frac{D}{2}}}{\Gamma\left(a_{1}\right) \Gamma\left(a_{2}\right)} \int_{0}^{\infty} d x d y x^{a_{1}-1} y^{a_{2}-1} \frac{\exp \left(y m^{2}\right)}{(x+y)^{\frac{D}{2}}} .
\end{aligned}
$$

After some algebra we get the MRE of this case:

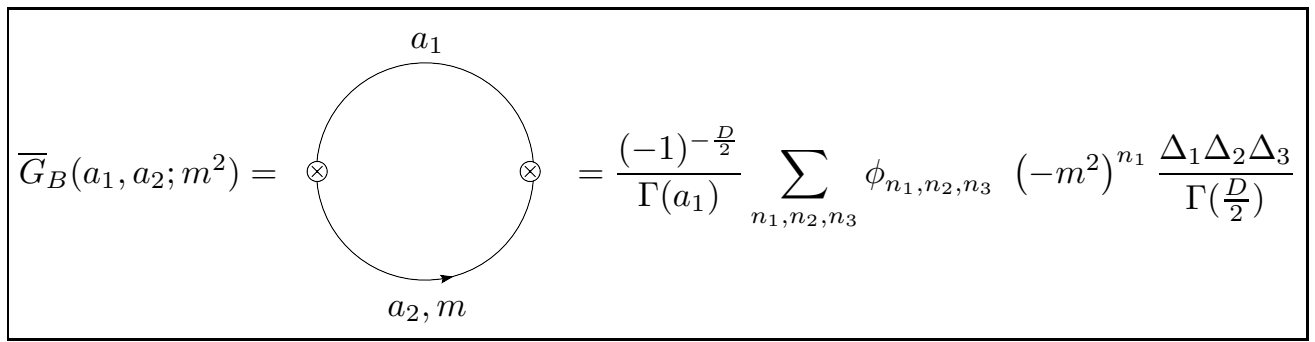


where:

$$
\left\{\begin{aligned}
\Delta_{1} & =\left\langle\frac{D}{2}+n_{2}+n_{3}\right\rangle, \\
\Delta_{2} & =\left\langle a_{1}+n_{2}\right\rangle, \\
\Delta_{3} & =\left\langle a_{2}+n_{1}+n_{3}\right\rangle .
\end{aligned}\right.
$$

Alternative II : Using the MRE of the massive propagator : The second alternative implies to extract the mass of the loop integral, that is, to find the MRE of the massive propagator. Then we have:

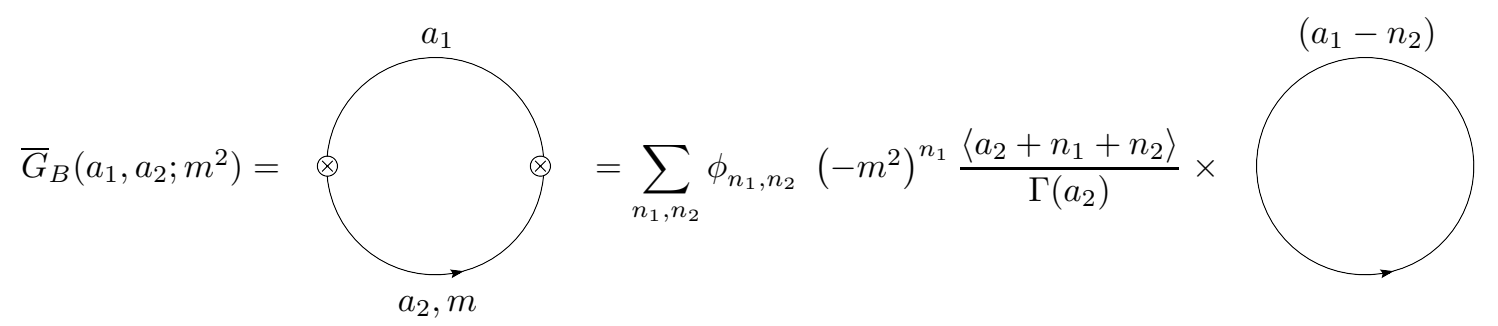

or equivalently:

$$
\bar{G}_{B}\left(a_{1}, a_{2} ; m^{2}\right)=\overbrace{a_{2}, m}^{a_{1}}=\frac{(-1)^{-\frac{D}{2}}}{\Gamma\left(a_{2}\right)} \sum_{n_{1}, n_{2}} \phi_{n_{1}, n_{2}}\left(-m^{2}\right)^{n_{1}} \frac{\left\langle a_{2}+n_{1}+n_{2}\right\rangle\left\langle a_{1}-n_{2}-\frac{D}{2}\right\rangle}{\Gamma\left(a_{1}-n_{2}\right)}
$$

Is evident that equation (82) is the most compact for function $\bar{G}_{B}$.

\subsubsection{Propagators with different masses : $\bar{G}_{D}$}

The next function is associated to a bubble with two propagators of different mass, which defines the loop function $\bar{G}_{D}$. Again we will deduce this function in two alternative ways, as was done before:

Alternative I : Expansion of the complete integral Applying Schwinger's parametrization allows to find the following structure for this module:

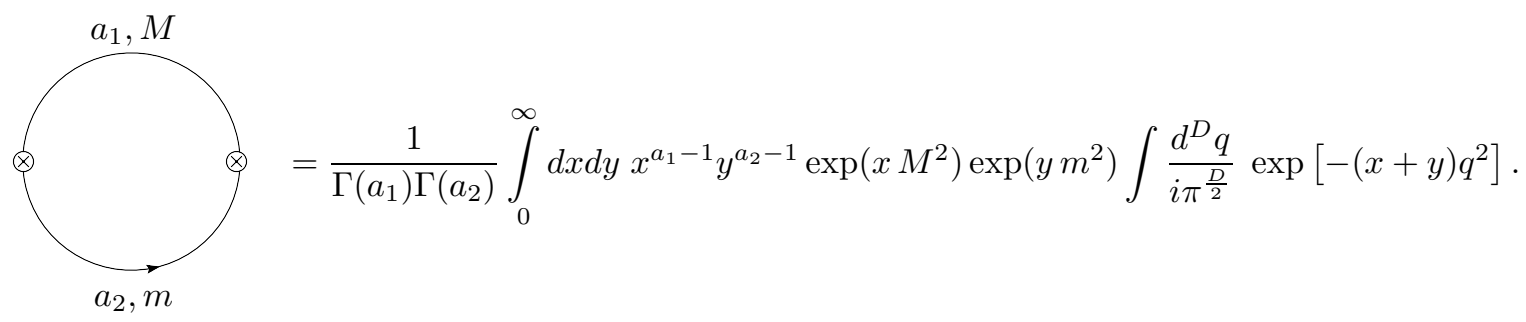

Performing the evaluations and replacements already described, we get the MRE for this case: 


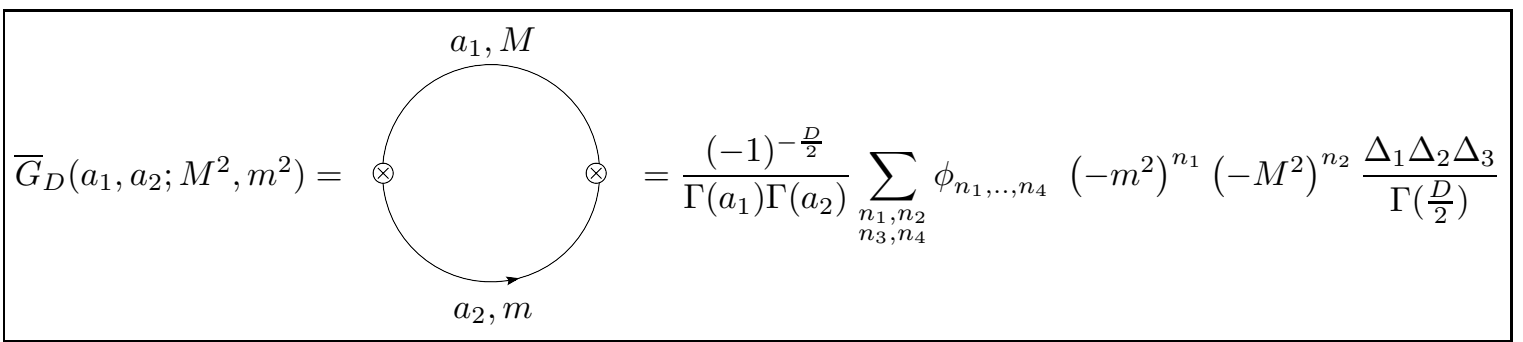

where the associated constraints are:

$$
\left\{\begin{aligned}
\Delta_{1} & =\left\langle\frac{D}{2}+n_{3}+n_{4}\right\rangle, \\
\Delta_{2} & =\left\langle a_{1}+n_{1}+n_{3}\right\rangle, \\
\Delta_{3} & =\left\langle a_{2}+n_{2}+n_{4}\right\rangle .
\end{aligned}\right.
$$

Alternative II : Expansion of massive propagators The momentum integral is given by:

$$
\bar{G}_{D}\left(a_{1}, a_{2} ; M^{2}, m^{2}\right)=\bigotimes_{a_{2}, m}^{a_{1}, M}=\int \frac{d^{D} q}{i \pi^{\frac{D}{2}}} \frac{1}{\left(q^{2}-M^{2}\right)^{a_{1}}\left(q^{2}-m^{2}\right)^{a_{2}}},
$$

then we expand each massive propagator:
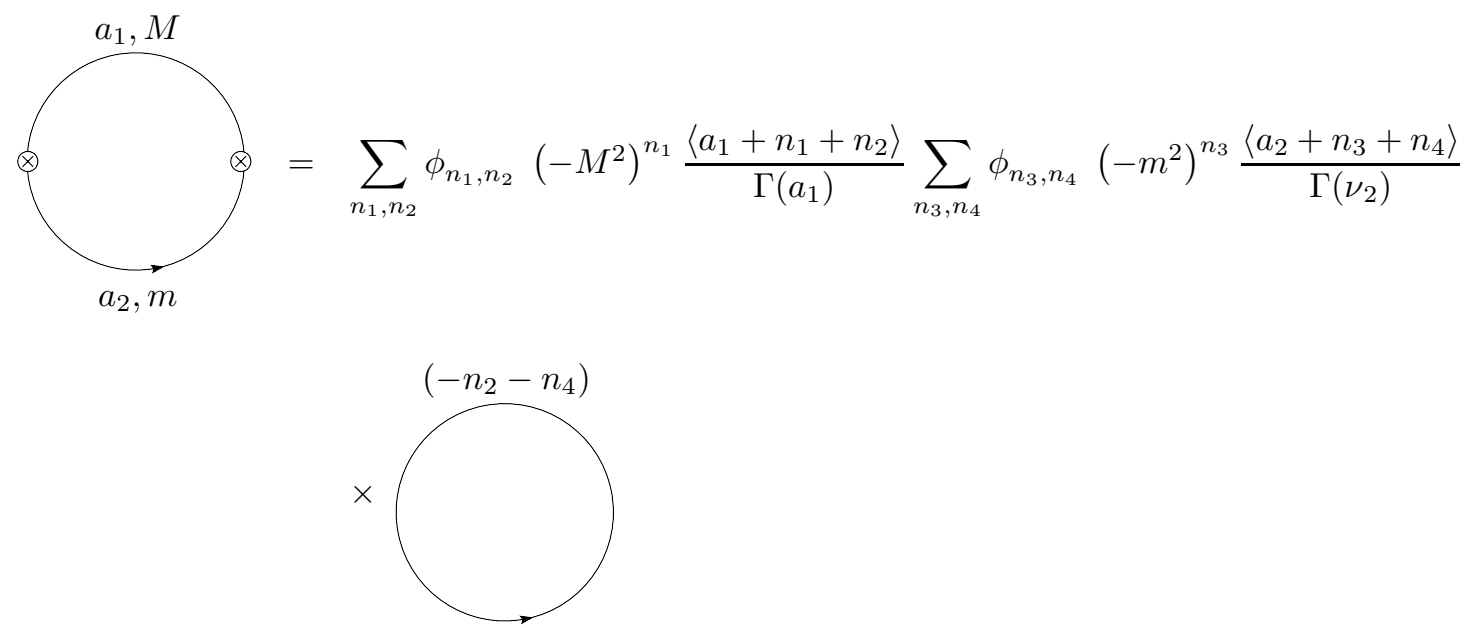

andDIDO using formula (61) we obtain the loop function $\bar{G}_{D}$ : 


$$
\begin{aligned}
\bar{G}_{D}\left(a_{1}, a_{2} ; M^{2}, m^{2}\right) & =\frac{(-1)^{-\frac{D}{2}}}{\Gamma\left(a_{1}\right) \Gamma\left(a_{2}\right)} \sum_{\substack{n_{1}, n_{2} \\
n_{3}, n_{4}}} \phi_{n_{1}, . ., n_{4}}\left(-M^{2}\right)^{n_{1}}\left(-m^{2}\right)^{n_{3}} \frac{\Delta_{1} \Delta_{2} \Delta_{3}}{\Gamma\left(-n_{2}-n_{4}\right)}
\end{aligned}
$$

with the following constraints:

$$
\left\{\begin{array}{l}
\Delta_{1}=\left\langle a_{1}+n_{1}+n_{2}\right\rangle, \\
\Delta_{2}=\left\langle a_{2}+n_{3}+n_{4}\right\rangle, \\
\Delta_{3}=\left\langle-n_{2}-n_{4}-\frac{D}{2}\right\rangle .
\end{array}\right.
$$

In all the massive cases we can use two equivalent ways of finding the MRE of the present module, but one of them will produce a mathematical expansion in a more reduced or minimal form. More compact MREs allow a considerable reduction of the possibilities of evaluating the sums of the expansion with the available Kronecker deltas. Remember that in the case of the MRE of a certain Feynman diagram, each alternative way of doing these evaluations generates a term (hypergeometric series) of the final solution, and therefore a minimal MRE allows to eliminate irrelevant or non existing terms (cases where it is not possible mathematically to perform the sum using the Kronecker deltas).

\section{Applications}

In this section we will use the previously obtained 1-loop functions and present three examples that will show and explain the IBFE methodology applied modularly. Together with this we will be able to visualize the advantages of this procedure compared with IBFE applied to the Schwinger's parametric representation of the complete diagram. In our previous work [4], we show as find explicitly solutions to start of the MRE equation of a Feynman diagram. In this work, we just compare the ways to obtain the respective MRE associated to a topology.

\subsection{Example I : Radiative correction to the three loop massless propagator}

Let us consider as a first example the three loop correction to the massless propagator, with momentum $p$, as shown in the graphical formula (90), applying the fractional expansion method loop by loop, and using the 1-loop functions previously obtained. In this case it is clear that we need only 1-loop functions of type $G_{A}$.

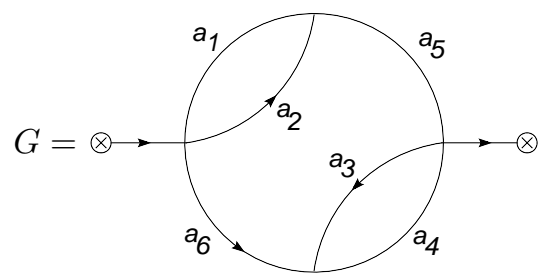

We start by reducing the two bubble insertions directly, using the pictorial formula (44), which allows us to get: 


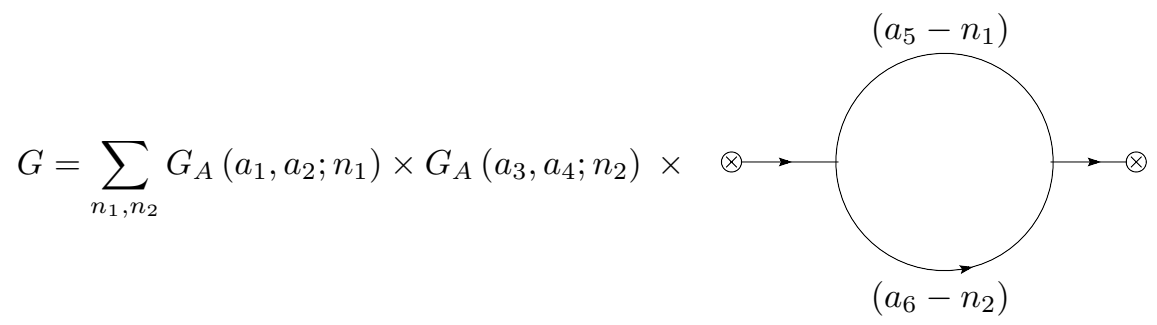

We can use (25) to sum the propagator indices, and then apply formula (44) for finally getting the diagram MRE in terms of a sum of products of 1-loop functions $G_{A}$ :

$$
G=\sum_{n_{1}, . ., n_{3}} G_{A}\left(a_{1}, a_{2} ; n_{1}\right) \times G_{A}\left(a_{3}, a_{4} ; n_{2}\right) \times G_{A}\left(a_{5}-n_{1}, a_{6}-n_{2} ; n_{3}\right) \times \otimes \stackrel{-n_{3}}{\longrightarrow} \otimes
$$

This result can be written also as:

$$
G=\sum_{n_{1}, . ., n_{3}} G_{A}\left(a_{1}, a_{2} ; n_{1}\right) \times G_{A}\left(a_{3}, a_{4} ; n_{2}\right) \times G_{A}\left(a_{5}-n_{1}, a_{6}-n_{2} ; n_{3}\right)\left(p^{2}\right)^{n_{3}},
$$

where the 1-loop functions $G_{A}$ for each case are determined, according to equation (42), by the following expression:

$$
G_{A}\left(a_{1}, a_{2} ; n_{1}\right)=\frac{(-1)^{-\frac{D}{2}}}{\Gamma\left(a_{1}\right) \Gamma\left(a_{2}\right)} \sum_{n_{4}, n_{5}} \phi_{n_{1}, n_{4}, n_{5}} \frac{\Delta_{1} \Delta_{2} \Delta_{3}}{\Gamma\left(\frac{D}{2}+n_{1}\right)},
$$

with constraints that are given by the identities:

$$
\left\{\begin{array}{l}
\Delta_{1}=\left\langle\frac{D}{2}+n_{1}+n_{4}+n_{5}\right\rangle \\
\Delta_{2}=\left\langle a_{1}+n_{1}+n_{4}\right\rangle \\
\Delta_{3}=\left\langle a_{2}+n_{1}+n_{5}\right\rangle
\end{array}\right.
$$

Similarly one has:

$$
\begin{gathered}
G_{A}\left(a_{3}, a_{4} ; n_{2}\right)=\frac{(-1)^{-\frac{D}{2}}}{\Gamma\left(a_{3}\right) \Gamma\left(a_{4}\right)} \sum_{n_{6}, n_{7}} \phi_{n_{2}, n_{6}, n_{7}} \frac{\Delta_{4} \Delta_{5} \Delta_{6}}{\Gamma\left(\frac{D}{2}+n_{2}\right)}, \\
\left\{\begin{array}{l}
\Delta_{4}=\left\langle\frac{D}{2}+n_{2}+n_{6}+n_{7}\right\rangle, \\
\Delta_{5}=\left\langle a_{3}+n_{2}+n_{6}\right\rangle, \\
\Delta_{6}=\left\langle a_{4}+n_{2}+n_{7}\right\rangle,
\end{array}\right.
\end{gathered}
$$

and finally:

$$
\begin{gathered}
G_{A}\left(a_{5}-n_{1}, a_{6}-n_{2} ; n_{3}\right)=\frac{(-1)^{-\frac{D}{2}}}{\Gamma\left(a_{5}-n_{1}\right) \Gamma\left(a_{6}-n_{2}\right)} \sum_{n_{8}, n_{9}} \phi_{n_{3}, n_{8}, n_{9}} \frac{\Delta_{7} \Delta_{8} \Delta_{9}}{\Gamma\left(\frac{D}{2}+n_{3}\right)}, \\
\left\{\begin{array}{l}
\Delta_{7}=\left\langle\frac{D}{2}+n_{3}+n_{8}+n_{9}\right\rangle, \\
\Delta_{8}=\left\langle a_{5}-n_{1}+n_{3}+n_{8}\right\rangle, \\
\Delta_{9}=\left\langle a_{6}-n_{2}+n_{3}+n_{9}\right\rangle .
\end{array}\right.
\end{gathered}
$$

Replacing these series in equation (93) allows to get the diagram MRE:

$$
G=\frac{(-1)^{-\frac{3 D}{2}}}{\prod_{i=1}^{4} \Gamma\left(a_{i}\right)} \sum_{n_{1}, . ., n_{9}} \phi_{n_{1}, . ., n_{9}} \frac{\left(p^{2}\right)^{n_{3}}}{\Gamma\left(\frac{D}{2}+n_{1}\right) \Gamma\left(\frac{D}{2}+n_{2}\right) \Gamma\left(\frac{D}{2}+n_{3}\right)} \frac{\prod_{j=1}^{9} \Delta_{j}}{\Gamma\left(a_{5}-n_{1}\right) \Gamma\left(a_{6}-n_{2}\right)}
$$


In order to show the advantages of modular IBFE, we show in Table I a comparison of the MRE obtained in this form with respect to that one that evaluates the complete diagram, that is which uses the parametric representation that incudes all loops simultaneously.

\begin{tabular}{lcc}
\hline & Complete (better factorization) & Modular \\
\hline Multiplicity multiregion series $(\sigma)$ & 11 & 9 \\
Kronecker deltas of the expansion $(\delta)$ & 11 & 9 \\
Multiplicity resulting series $(\sigma-\delta)$ & 0 & 0 \\
Posible contributions to the solution $\left(C_{\delta}^{\sigma}\right)$ & 1 & 1 \\
\hline
\end{tabular}

(Table I)

The expression (100) is equivalent to equation (29), which was deduced for the same diagram but using the usual 1-loop function (23). The first conclusion is that the fractional expansion technique is, for cases of loop by loop reducible massless diagrams, more tedious than the conventional. In spite of this, we will see that for cases in which the diagram contains different mass scales, the present technique es very powerful, and this will become clear in the following example.

\subsection{Example II : Propagator with two mass scales and two loops}

Let us consider a graph which consists of a two loop propagator with two different mass scales, as shown in the figure:

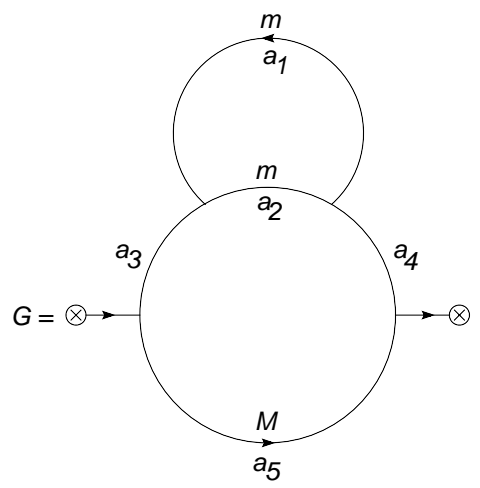

We first reduce the loop associated to the mass $m$, which uses the 1-loop function $G_{C}$ (equation (56)), and then we get:

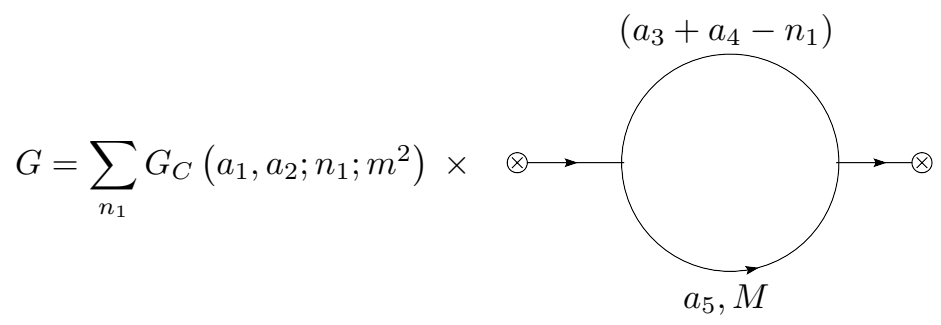

For the resulting loop reduction we use formula (48), obtaining the massive diagram MRE:

$$
G=\sum_{n_{1}, n_{2}} G_{C}\left(a_{1}, a_{2} ; n_{1} ; m^{2}\right) \times G_{B}\left(a_{5}, a_{3}+a_{4}-n_{1} ; n_{2} ; M^{2}\right) \times \otimes \stackrel{-n_{2}}{\longrightarrow} \otimes
$$

which is equivalent to the algebraic expression: 


$$
G=\sum_{n_{1}, n_{2}} G_{C}\left(a_{1}, a_{2} ; n_{1} ; m^{2}\right) \times G_{B}\left(a_{5}, a_{3}+a_{4}-n_{1} ; n_{2} ; M^{2}\right)\left(p^{2}\right)^{n_{2}} .
$$

The 1-loop functions $G_{C}$ and $G_{B}$ in (104) are given by:

$$
G_{C}\left(a_{1}, a_{2} ; n_{1} ; m^{2}\right)=\frac{(-1)^{-\frac{D}{2}}}{\Gamma\left(a_{1}\right) \Gamma\left(a_{2}\right)} \sum_{n_{3}, n_{4}, n_{5}} \phi_{n_{1}, n_{3}, n_{4}, n_{5}}\left(-m^{2}\right)^{n_{3}} \frac{\Delta_{1} \Delta_{2} \Delta_{3}}{\Gamma\left(\frac{D}{2}+n_{1}-n_{3}\right)},
$$

where the constraints are:

$$
\left\{\begin{array}{l}
\Delta_{1}=\left\langle\frac{D}{2}+n_{1}-n_{3}+n_{4}+n_{5}\right\rangle \\
\Delta_{2}=\left\langle a_{1}+n_{1}+n_{4}\right\rangle \\
\Delta_{3}=\left\langle a_{2}+n_{1}+n_{5}\right\rangle
\end{array}\right.
$$

and:

$$
G_{B}\left(a_{5}, a_{3}+a_{4}-n_{1} ; n_{2} ; M^{2}\right)=\frac{(-1)^{-\frac{D}{2}}}{\Gamma\left(a_{5}\right) \Gamma\left(a_{3}+a_{4}-n_{1}\right)} \sum_{n_{6}, n_{7}, n_{8}} \phi_{n_{2}, n_{6}, n_{7}, n_{8}}\left(-M^{2}\right)^{n_{6}} \frac{\Delta_{4} \Delta_{5} \Delta_{6}}{\Gamma\left(\frac{D}{2}+n_{2}\right)},
$$

with the constraints:

$$
\left\{\begin{array}{l}
\Delta_{4}=\left\langle\frac{D}{2}+n_{2}+n_{7}+n_{8}\right\rangle \\
\Delta_{5}=\left\langle a_{5}+n_{2}+n_{6}+n_{7}\right\rangle \\
\Delta_{6}=\left\langle a_{3}+a_{4}-n_{1}+n_{2}+n_{8}\right\rangle .
\end{array}\right.
$$

The replacement in (104) allows to finally get the MRE of diagram $G$ :

$$
G=\frac{(-1)^{-D}}{\Gamma\left(a_{1}\right) \Gamma\left(a_{2}\right) \Gamma\left(a_{5}\right)} \sum_{n_{1}, . ., n_{8}} \phi_{n_{1}, . ., n_{8}} \frac{\left(p^{2}\right)^{n_{2}}\left(-m^{2}\right)^{n_{3}}\left(-M^{2}\right)^{n_{6}}}{\Gamma\left(\frac{D}{2}+n_{1}-n_{3}\right) \Gamma\left(\frac{D}{2}+n_{2}\right)} \frac{\Delta_{1} \Delta_{2} \Delta_{3} \Delta_{4} \Delta_{5} \Delta_{6}}{\Gamma\left(a_{3}+a_{4}-n_{1}\right)}
$$

Operationally it is not possible to apply directly the conventional 1-loop reduction (23) to this massive example, as has been done here with the fractionally expanded 1-loop functions. Moreover, it is also possible to verify that the diagram MRE obtained loop by loop is far more direct and simple than to get this expansion considering all loops simultaneously. This can be seen from Table II, where we observe that the modular application of the IBFE technique not only reduces the MRE of the diagram in terms of sums and deltas, but also brings in a significant reduction of the number of irrelevant terms and therefore the calculation time of the solution is optimized.

\begin{tabular}{lcc}
\hline & Complete (better factorization) & Modular \\
\hline Multiplicity multiregion series $(\sigma)$ & 11 & 8 \\
Kronecker deltas of the expansion $(\delta)$ & 9 & 6 \\
Multiplicity resulting series $(\sigma-\delta)$ & 2 & 2 \\
Possible contributions to the solution $\left(C_{\delta}^{\sigma}\right)$ & 55 & 28 \\
\hline
\end{tabular}

$($ Tabla $I I)$

\subsection{Example III : Vacuum fluctuation with three mass scales and five loops}

The next diagram that we will analyze is one without external lines, composed of five loops and three different mass scales $\left\{m_{1}, m_{2}, M\right\}$, arranged as shown here: 


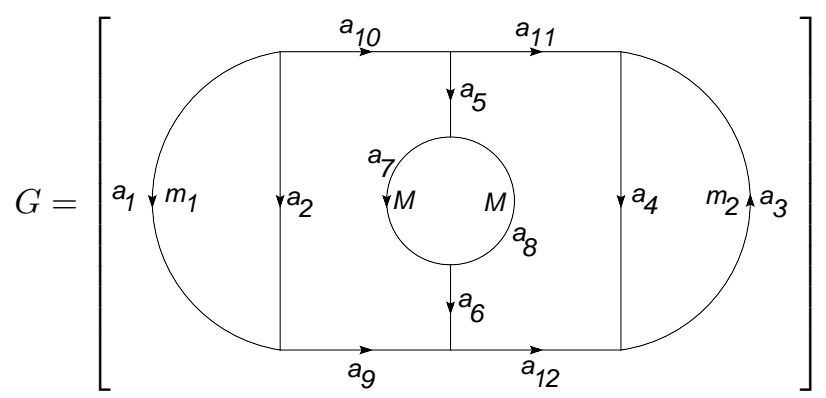

In the first step we reduce the left and right massive loops in terms of the 1-loop functions $G_{B}$ :

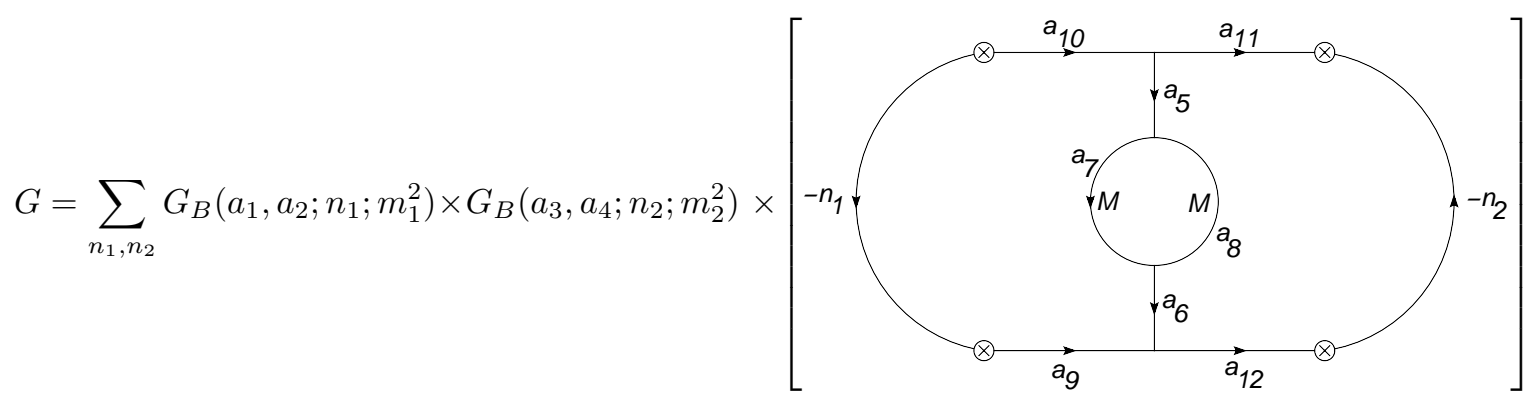

Applying formula (25) in order to sum the propagator indices, we get the equivalent equation:

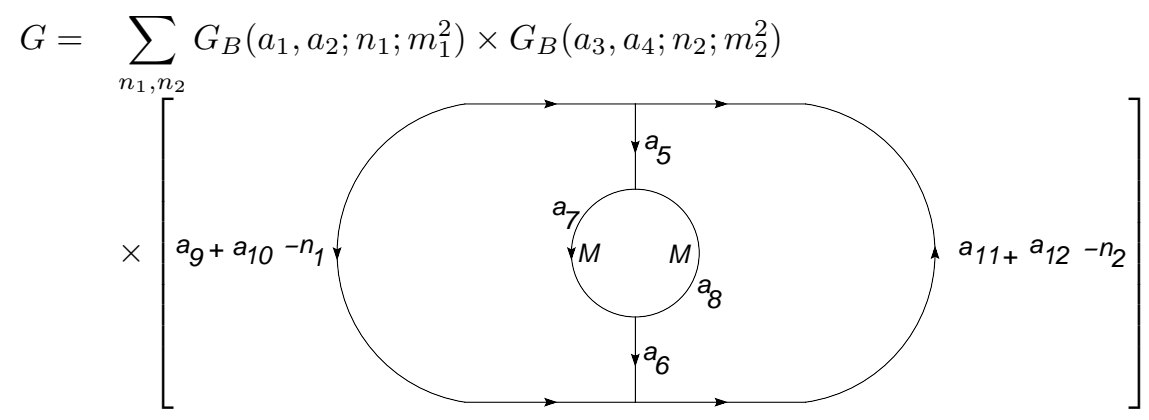

Then we eliminate the internal bubble:

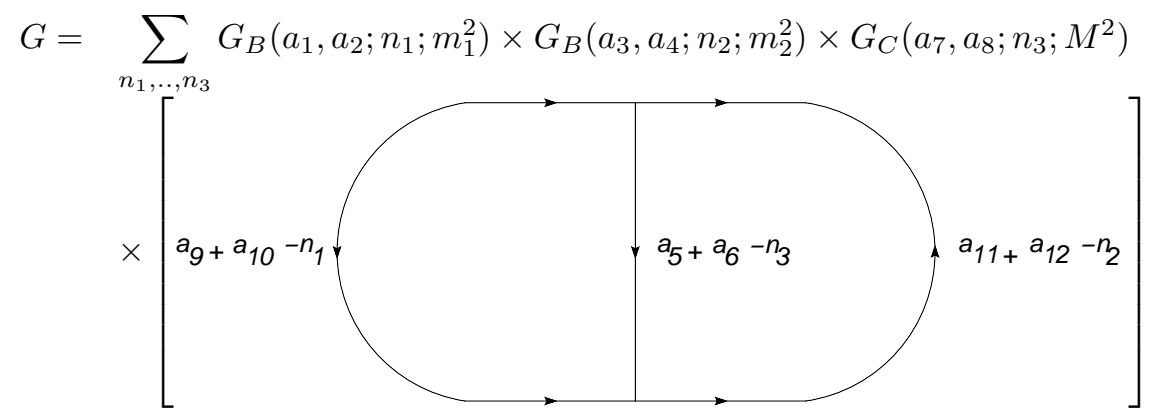

continuing with a reduction of the left loop: 


$$
G=\sum_{n_{1}, . ., n_{4}} G_{B}\left(a_{1}, a_{2} ; n_{1} ; m_{1}^{2}\right) \times G_{B}\left(a_{3}, a_{4} ; n_{2} ; m_{2}^{2}\right) \times G_{C}\left(a_{7}, a_{8} ; n_{3} ; M^{2}\right)
$$

The last loop reduction is represented by the function $\bar{G}_{A}$, which determines finally the MRE of the diagram:

$$
\begin{aligned}
G= & \sum_{n_{1}, . ., n_{4}} G_{B}\left(a_{1}, a_{2} ; n_{1} ; m_{1}^{2}\right) \times G_{B}\left(a_{3}, a_{4} ; n_{2} ; m_{2}^{2}\right) \times G_{C}\left(a_{7}, a_{8} ; n_{3} ; M^{2}\right) \times G_{A}\left(a_{9,10}-n_{1}, a_{5,6}-n_{3} ; n_{4}\right) \\
& \times \bar{G}_{A}\left(a_{11,12}-n_{2},-n_{4}\right) .
\end{aligned}
$$

The corresponding 1-loop functions in this example are given by:

$$
\begin{aligned}
& G_{B}\left(a_{1}, a_{2} ; n_{1} ; m_{1}^{2}\right)=\frac{(-1)^{-\frac{D}{2}}}{\Gamma\left(a_{1}\right) \Gamma\left(a_{2}\right)} \sum_{n_{5}, . ., n_{7}} \phi_{n_{1}, n_{5}, . ., n_{7}} \frac{\left(-m_{1}^{2}\right)^{n_{7}}}{\Gamma\left(\frac{D}{2}+n_{1}\right)} \Delta_{1} \Delta_{2} \Delta_{3}, \\
& \left\{\begin{array}{l}
\Delta_{1}=\left\langle a_{1}+n_{1}+n_{5}+n_{7}\right\rangle, \\
\Delta_{2}=\left\langle a_{2}+n_{1}+n_{6}\right\rangle, \\
\Delta_{3}=\left\langle\frac{D}{2}+n_{1}+n_{5}+n_{6}\right\rangle,
\end{array}\right. \\
& G_{B}\left(a_{3}, a_{4} ; n_{2} ; m_{2}^{2}\right)=\frac{(-1)^{-\frac{D}{2}}}{\Gamma\left(a_{3}\right) \Gamma\left(a_{4}\right)} \sum_{n_{8}, . ., n_{10}} \phi_{n_{2}, n_{8}, . ., n_{10}} \frac{\left(-m_{2}^{2}\right)^{n_{10}}}{\Gamma\left(\frac{D}{2}+n_{2}\right)} \Delta_{4} \Delta_{5} \Delta_{6}, \\
& \left\{\begin{array}{l}
\Delta_{4}=\left\langle a_{3}+n_{2}+n_{8}+n_{10}\right\rangle, \\
\Delta_{5}=\left\langle a_{4}+n_{2}+n_{9}\right\rangle \\
\Delta_{6}=\left\langle\frac{D}{2}+n_{2}+n_{8}+n_{9}\right\rangle,
\end{array}\right. \\
& G_{C}\left(a_{7}, a_{8} ; n_{3} ; M^{2}\right)=\frac{(-1)^{-\frac{D}{2}}}{\Gamma\left(a_{7}\right) \Gamma\left(a_{8}\right)} \sum_{n_{11}, . ., n_{13}} \phi_{n_{3}, n_{11}, . ., n_{13}} \frac{\left(-M^{2}\right)^{n_{13}}}{\Gamma\left(\frac{D}{2}+n_{3}-n_{13}\right)} \Delta_{7} \Delta_{8} \Delta_{9}, \\
& \left\{\begin{aligned}
\Delta_{7} & =\left\langle a_{7}+n_{3}+n_{11}\right\rangle, \\
\Delta_{8} & =\left\langle a_{8}+n_{3}+n_{12}\right\rangle, \\
\Delta_{9} & =\left\langle\frac{D}{2}+n_{3}-n_{13}+n_{11}+n_{12}\right\rangle,
\end{aligned}\right. \\
& G_{A}\left(a_{9,10}-n_{1}, a_{5,6}-n_{3} ; n_{4}\right)=\frac{(-1)^{-\frac{D}{2}}}{\Gamma\left(a_{9}+a_{10}-n_{1}\right) \Gamma\left(a_{5}+a_{6}-n_{3}\right)} \sum_{n_{14}, n_{15}} \phi_{n_{4}, n_{14}, n_{15}} \frac{\Delta_{10} \Delta_{11} \Delta_{12}}{\Gamma\left(\frac{D}{2}+n_{4}\right)} \\
& \left\{\begin{array}{l}
\Delta_{10}=\left\langle\frac{D}{2}+n_{4}+n_{14}+n_{15}\right\rangle, \\
\Delta_{11}=\left\langle a_{9}+a_{10}-n_{1}+n_{4}+n_{14}\right\rangle, \\
\Delta_{12}=\left\langle a_{5}+a_{6}-n_{3}+n_{4}+n_{15}\right\rangle,
\end{array}\right. \\
& \bar{G}_{A}\left(a_{11}+a_{12}-n_{2},-n_{4}\right)=(-1)^{-\frac{D}{2}} \frac{\Delta_{13}}{\Gamma\left(a_{11}+a_{12}-n_{2}-n_{4}\right)}, \\
& \left\{\Delta_{13}=\left\langle a_{11}+a_{12}-n_{2}-n_{4}-\frac{D}{2}\right\rangle .\right.
\end{aligned}
$$

Finally we obtain the MRE associated to the diagram: 


$$
\begin{aligned}
G= & \frac{(-1)^{-\frac{5 D}{2}}}{\Gamma\left(a_{7}\right) \Gamma\left(a_{8}\right) \prod_{j=1}^{4} \Gamma\left(a_{j}\right)} \sum_{n_{1}, . ., n_{15}} \phi_{n_{1}, . ., n_{17}}\left(-m_{1}^{2}\right)^{n_{7}}\left(-m_{2}^{2}\right)^{n_{10}}\left(-M^{2}\right)^{n_{13}} \\
& \frac{1}{\Gamma\left(\frac{D}{2}+n_{1}\right) \Gamma\left(\frac{D}{2}+n_{2}\right) \Gamma\left(\frac{D}{2}+n_{3}-n_{13}\right) \Gamma\left(\frac{D}{2}+n_{4}\right) \Gamma\left(\frac{D}{2}\right)} \\
& \frac{\prod_{k=1}^{13} \Delta_{k}}{\Gamma\left(a_{9}+a_{10}-n_{1}\right) \Gamma\left(a_{5}+a_{6}-n_{3}\right) \Gamma\left(a_{11}+a_{12}-n_{2}-n_{4}\right)}
\end{aligned}
$$

Table III shows again a comparison of the IBFE applied modularly or complete to the diagram.

\begin{tabular}{lcc}
\hline & Complete (better factorization) & Modular \\
\hline Multiplicity multiregion series $(\sigma)$ & 21 & 15 \\
Kronecker deltas of the expansion $(\delta)$ & 19 & 13 \\
Multiplicity resulting series $(\sigma-\delta)$ & 2 & 2 \\
Possible contributions to the solution $\left(C_{\delta}^{\sigma}\right)$ & 210 & 105 \\
\hline
\end{tabular}

(Table III)

\section{Comments}

\subsection{Other 1-loop functions associated to the bubble module}

The 1-loop functions for diagrams that contain the bubble type insertions previously deduced do not contemplate all the possible cases. To see this let us try to solve the following topology:

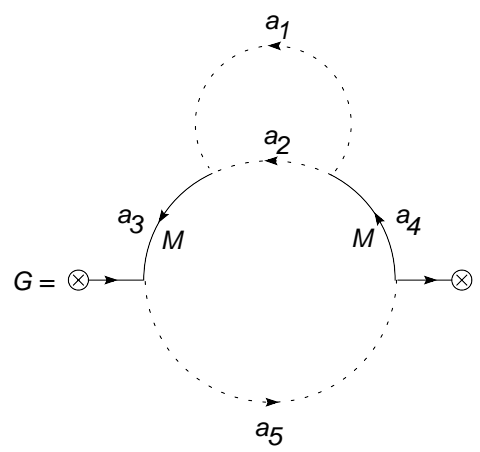

This diagram contains two massive propagators, each of them characterized by mass $M$, arranged as shown in the figure (continuous line), and the rest of the propagators are massless (dashed lines). It can be easily evaluated if Schwinger's parametric representation of the whole diagram is integrated. Let us see:

$$
G=\frac{(-1)^{-D}}{\prod_{j=1}^{5} \Gamma\left(a_{j}\right)} \int_{0}^{\infty} d \vec{x} \frac{\exp \left(\left(x_{3}+x_{4}\right) M^{2}\right) \exp \left(-\frac{\left[x_{1} x_{2}+\left(x_{1}+x_{2}\right)\left(x_{3}+x_{4}\right)\right] x_{5}}{x_{1} x_{2}+\left(x_{1}+x_{2}\right)\left(x_{3}+x_{4}\right)+x_{5}\left(x_{3}+x_{4}\right)} p^{2}\right)}{\left[x_{1} x_{2}+\left(x_{1}+x_{2}\right)\left(x_{3}+x_{4}\right)+x_{5}\left(x_{3}+x_{4}\right)\right]^{\frac{D}{2}}} .
$$

The integral is written in a factorized form and it is readily evaluated with IBFE. After doing the corresponding expansions we realize that the obtained MRE has ten summations and nine deltas, so the solutions will correspond to one variable hypergeometric functions $\left({ }_{q} F_{q-1}\right)$, which is something expected given the type of topology and the number of energy scales present in the problem. Moreover, we will have at most ten possible contributions that distribute themselves in the kinematical regions $\left|\frac{M^{2}}{p^{2}}\right|<1$ and $\left|\frac{p^{2}}{M^{2}}\right|<1$. 
Now we will try to solve this diagram, but this time applying the 1-loop functions deduced in this chapter. The upper loop is easily reduced using the 1-loop function $G_{A}$, getting then the following reduced diagram:

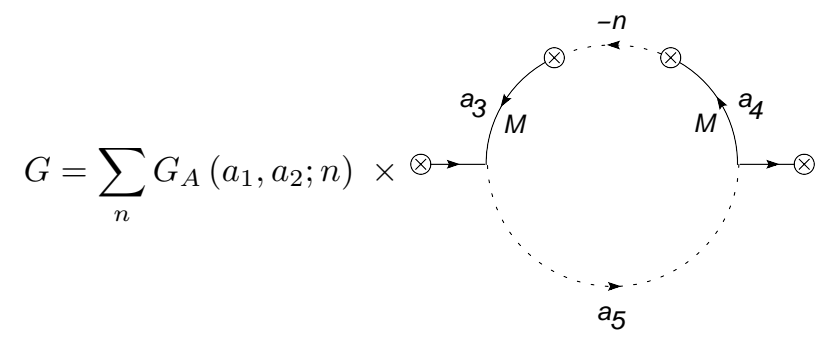

Nevertheless, we obtain one loop which is not possible to reduce with the 1-loop functions previously defined. The reason is the presence of the mixed massive propagator in the upper branch of the diagram. Thus it is necessary to consider a new loop function that we will call $G_{E}$, and which is associated to the generic topology of the form:

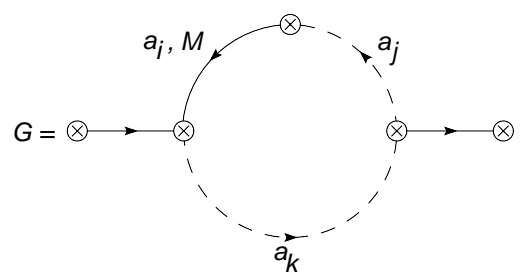

Let us try to find the MRE for this new loop function. For this we start with the corresponding Schwinger parametric representation.

$$
G=\frac{(-1)^{-\frac{D}{2}}}{\Gamma\left(a_{i}\right) \Gamma\left(a_{j}\right) \Gamma\left(a_{k}\right)} \int_{0}^{\infty} d \vec{x} \frac{\exp \left(x_{i} M^{2}\right) \exp \left(-\frac{\left(x_{i}+x_{j}\right) x_{k}}{x_{i}+x_{j}+x_{k}} p^{2}\right)}{\left(x_{i}+x_{j}+x_{k}\right)^{\frac{D}{2}}} .
$$

When expanding the exponential that contains $p^{2}$ the following series is obtained:

$$
G=\sum_{n} G_{E}\left(a_{i}, a_{j}, a_{k} ; n ; M^{2}\right) \frac{1}{\left(p^{2}\right)^{-n}}
$$

and just as before the 1-loop function is defined:

$$
G_{E}\left(a_{i}, a_{j}, a_{k} ; n ; M^{2}\right)=\frac{(-1)^{-\frac{D}{2}}}{\Gamma\left(a_{i}\right) \Gamma\left(a_{j}\right) \Gamma\left(a_{k}\right)} \phi_{n} \int_{0}^{\infty} d \vec{x} \frac{\exp \left(x_{i} M^{2}\right) x_{k}^{n}\left(x_{i}+x_{j}\right)^{n}}{\left(x_{i}+x_{j}+x_{k}\right)^{\frac{D}{2}+n}} .
$$

Taking into account the complete expansion procedure finally one finds that the multiregion series for this new 1-loop function is:

$$
G_{E}\left(a_{i}, a_{j}, a_{k} ; n ; M^{2}\right)=\frac{(-1)^{-\frac{D}{2}}}{\Gamma\left(a_{i}\right) \Gamma\left(a_{j}\right) \Gamma\left(a_{k}\right)} \sum_{s_{1}, ., s_{5}} \phi_{n, s_{1}, \ldots, s_{5}}\left(-M_{j}^{2}\right)^{s_{1}} \frac{\Delta_{1} \ldots \Delta_{5}}{\Gamma\left(\frac{D}{2}+n\right) \Gamma\left(-n-s_{2}\right)}
$$

with the constraints:

$$
\left\{\begin{aligned}
\Delta_{1} & =\left\langle\frac{D}{2}+n+s_{2}+s_{3}\right\rangle, \\
\Delta_{2} & =\left\langle-n-s_{2}+s_{4}+s_{5}\right\rangle, \\
\Delta_{3} & =\left\langle a_{i}+s_{1}+s_{4}\right\rangle, \\
\Delta_{4} & =\left\langle a_{j}+s_{5}\right\rangle \\
\Delta_{5} & =\left\langle a_{k}+n+s_{3}\right\rangle .
\end{aligned}\right.
$$


Graphically this result can be represented as follows:

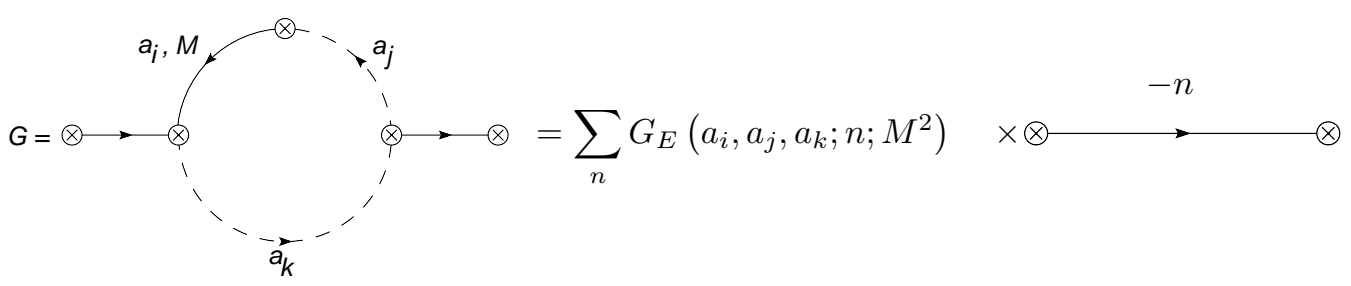

This loop contributes with $6 \Sigma$ and $5 \delta$. Coming back now to our initial problem presented in equation (125), and using formula (134) we find the MRE for this problem:

$$
G=\sum_{n, l} G_{A}\left(a_{1}, a_{2} ; n\right) \times G_{E}\left(a_{3}+a_{4},-n, a_{5} ; l ; M^{2}\right)\left(p^{2}\right)^{l},
$$

which can be compared with the equivalent MRE coming from the parametric representation of the complete diagram (Table IV):

\begin{tabular}{lcc}
\hline & Complete (better factorization) & Modular \\
\hline Multiplicity multiregion series $(\sigma)$ & 10 & 9 \\
Kronecker deltas of the expansion $(\delta)$ & 9 & 8 \\
Multiplicity resultanting series $(\sigma-\delta)$ & 1 & 1 \\
Possible contributions to the solution $\left(C_{\delta}^{\sigma}\right)$ & 10 & 9 \\
\hline
\end{tabular}

(Table IV)

The first we should notice is that in this case once again the expression for the MRE obtained in terms of modules is more reduced than the similar expression which evaluates the complete diagram. A second aspect is that just as the loop function $G_{E}$, there are many others which are variations of the bubble module and that have not been included here, although as has been done above any other configuration has an MRE which can be trivially found.

\subsection{Generic diagrams with bubble type insertions}

We have shown that the IBFE technique is very useful and simple to apply in diagrams that are built by successive one loop insertions, which can be either massless or massive. Nevertheless, the modular reduction of a diagram using the IBFE technique is not only readily applicable to this type of topologies, but as will be discussed later, the previously deduced formulae are also useful for reducing any bubble type subgraph that is contained within a generic graph. As an example let us analyze the following diagram:

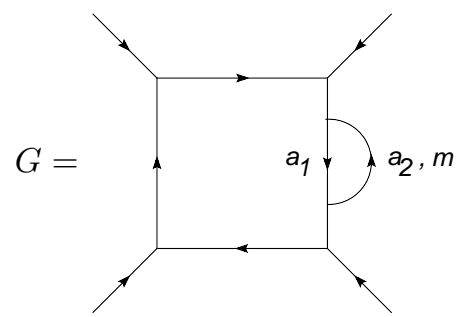

Independently of the kinematic characteristics of this diagram, our interest is in the propagator correction with a bubble type insertion and with one of the propagators with mass $m$. Using the respective loop function in order to reduce the loop we obtain the following graphical equation: 


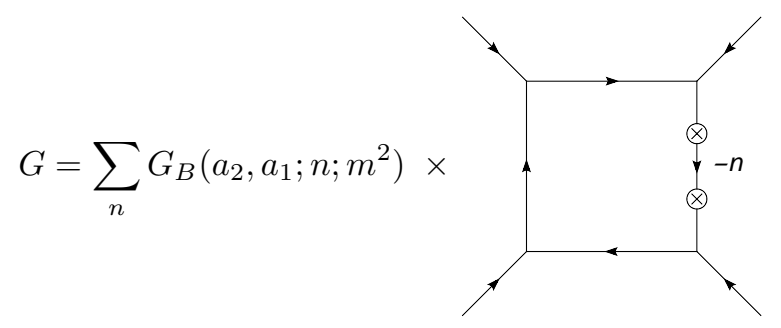

From a topological point of view one can see that there is a reduction, so to find the MRE of this resulting one loop diagram is simpler than the original two loop one. Just as in this example, it is possible to use the 1-loop functions in generic diagrams whose propagators contain bubble type corrections and its variations, which simplifies considerably the search of an MRE of certain diagrams and its solutions.

\section{3 n-loop modules and $n$-loop functions}

We have shown that it is possible to apply IBFE to loop by loop recursively built subgraphs and find the respective MRE in terms of the 1-loop functions defined above. Nevertheless, the flexibility of IBFE goes beyond that, and it is possible to generate also $n$-loop functions or equivalently module functions that consider two or more loops simultaneously. Such a module, with $n$ loops, will lead to a growing number of $n$-loop functions when one takes into account the different possibilities of distributing the propagator masses of the $n$-loop module. In general to work modularly is advantageous for reducing diagrams, since it simplifies the search of the MRE. Nevertheless, if the idea is to have less algebraic manipulations and a minimal expression for the MRE of the topology, then the previous examples indicate that the MRE is going to be minimal when the modules under consideration are compositions of only one loop.

\section{Conclusions}

In this work we have described an efficient and easy way to to implement the integration technique IBFE, applied to a specific Feynman diagram. The result that has been reached using the concept of modular reduction has two important characteristics: first, in general the diagram multiregion expansion of a given diagram is shorter than if the MRE is obtained from the parametric representation of the complete diagram; second, the modular treatment operationally facilitates obtaining the multiregion expansion. Let us discuss this last point, comparing the modular reduction in cases of loop by loop reducible diagrams with 1-loop insertions in a massless theory which are evaluated using conventional calculation. The difference is that with IBFE it is possible to do a calculation with the same degree of simplicity, although for more complex bubble type diagrams it is now possible to include masses.

The modular reduction idea leads in general to a minimization of the MRE of a diagram, which is more evident when the modules to reduce are composed of one loop. In fact, the loop by loop reduction optimizes the IBFE application. In more general terms and beyond this work, depending on the one loop topology the minimal MRE is reached by a systematic reduction going from the topologically simplest to the more complex: bubbles, Triangles, Boxes, etc.

The importance of a minimal MRE is of course tied to a minimization of the time of finding the solutions, since the MRE gets the solutions to the parametric integrals evaluating summations with Kronecker deltas in all possible combinations, and now the MRE is shorter (less summations and Kronecker deltas, which diminish equally), then there are less combinations that need to be taken into account in order to find the solution. Basically what happens is that several combinations which do not lead to relevant terms in the solution are eliminated, and given that with IBFE the integration is replaced by a linear system evaluation, the minimized MRE implies smaller linear systems, and therefore simpler to analyze.

The integration technique IBFE is a simple method and at the same time powerful, which can be advantageously compared with other Feynman diagram evaluation, and which does not require great mathematical knowledge. Although here we have concentrated in a scalar theory, this method is directly applicable to other theories by previous scalarization of the tensorial loop integrals, which produces a sum of scalar integrals. 
In general the most complicated case of an $N$ propagator tensorial integral is precisely the corresponding $N$ propagator scalar integral. This is the reason for improving or creating numerical or analytical techniques for the evaluation of scalar integrals in perturbative studies in field theory.

Acknowledgements : We acknowledge support from Fondecyt (Chile) under Grant No. 3080029 and Grant Fund. Andes No. C-14055/20. 


\section{A Summary of formulae for the bubble module}

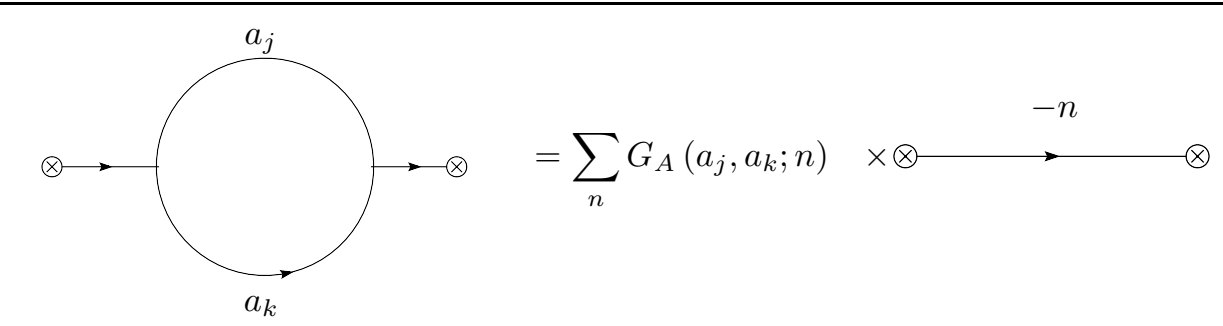

Composition $\Longrightarrow 3 \Sigma / 3 \delta$.
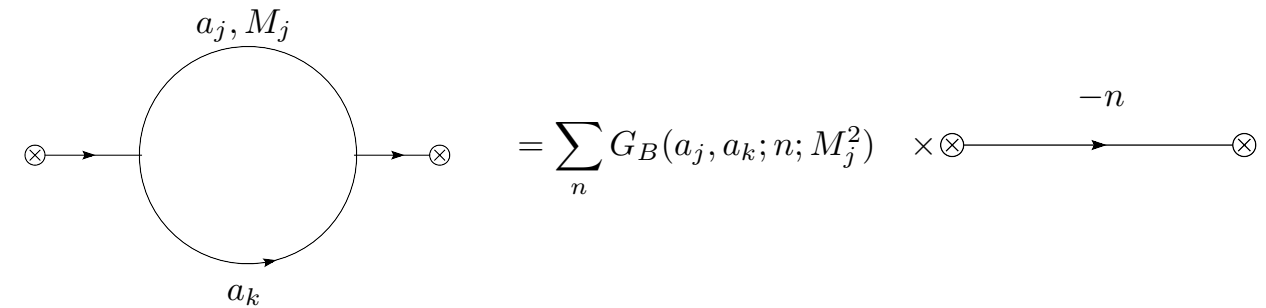

Composition $\Longrightarrow 4 \Sigma / 3 \delta$.

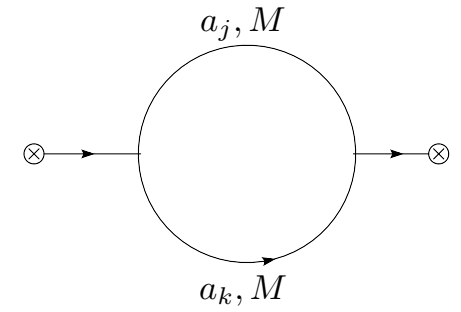

$=\sum_{n} G_{C}\left(a_{j}, a_{k} ; n ; M^{2}\right)$

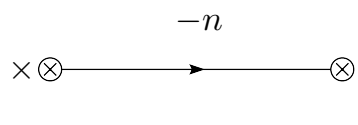

Composition $\Longrightarrow 4 \Sigma / 3 \delta$.

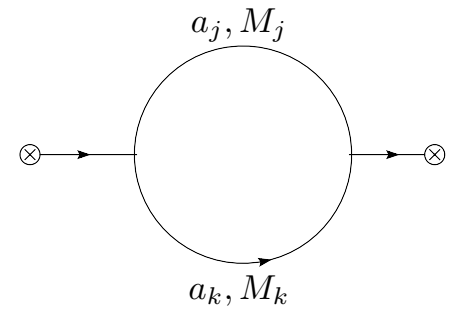

$=\sum_{n} G_{D}\left(a_{j}, a_{k} ; n ; M_{j}^{2}, M_{k}^{2}\right)$

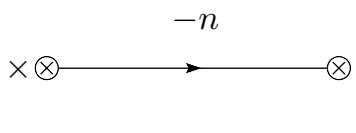

Composition $\Longrightarrow 5 \Sigma / 3 \delta$. 


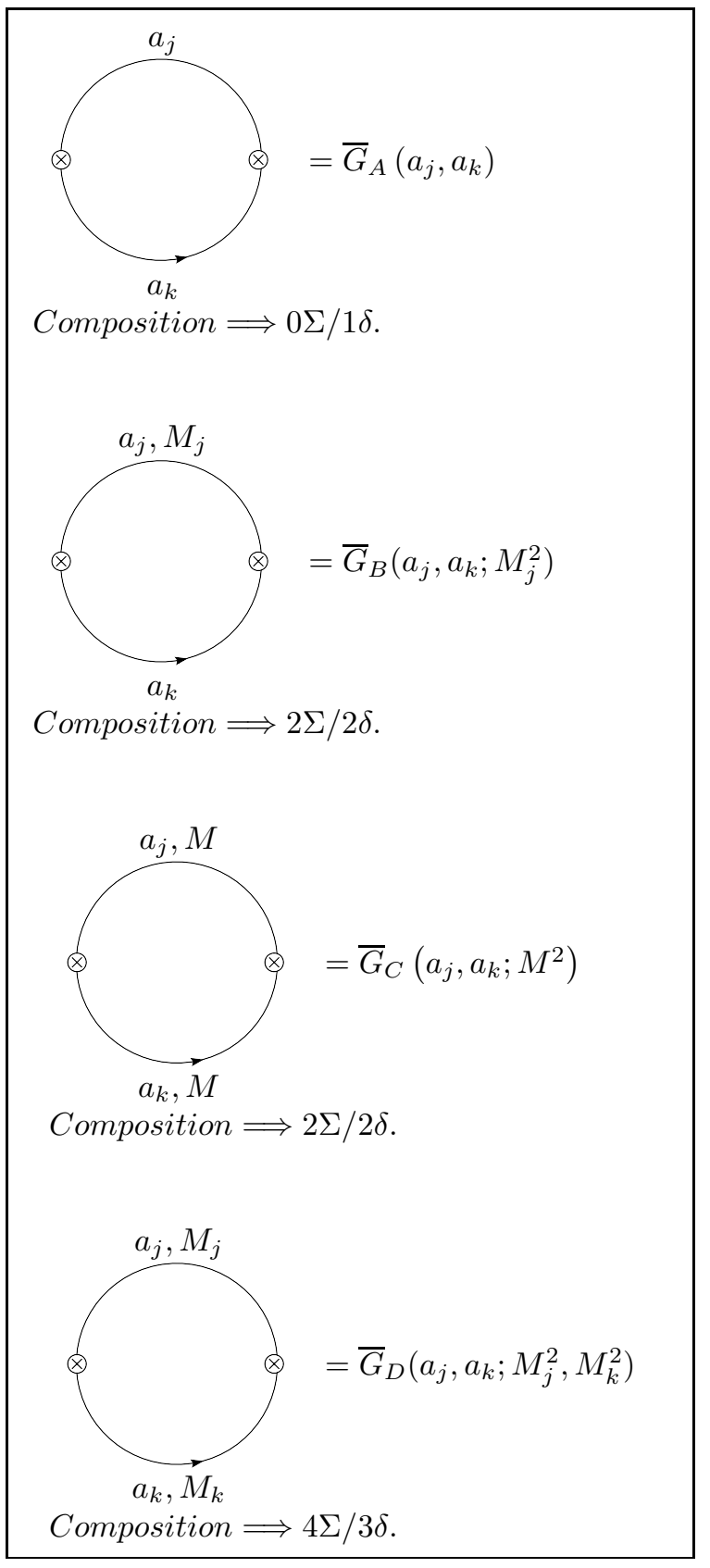

\section{B Hypergeometric functions}

In the evaluation of $L$ loop Feynman diagrams, all the possible solutions that can be found are presented in terms of multivariable generalized hypergeometric series. The actual value of the variables is given in general by ratios of two energy scales associated to the graph [4], including also arguments of value ' 1 '. The purpose of this appendix is to provide the necessary information about simple and double hypergeometric functions $[17,18,19,20,21]$, which appear in the solution of the loop integrals, and in particular present their convergence conditions, which are what finally define the different kinematical regions associated to the solution of a specific Feynman diagram. 


\section{B.1 Definition of the generalized hypergeometric function}

The functions associated to one variable series solutions of a Feynman diagram are always expressible in terms of generalized hypergeometric functions, in particular the hypergeometric function of order $(q, q-1)$, which is the only one variable series that is solution to the loop integrals. Thsi function is conventionally denoted as:

$$
{ }_{q} F_{q-1}(\{a\} ;\{b\} ; z) \equiv{ }_{q} F_{q-1}\left(\begin{array}{c}
\{a\} \\
\{b\}
\end{array} \mid z\right) \equiv \sum_{k=0}^{\infty} \frac{\left(a_{1}\right)_{k} \ldots\left(a_{q}\right)_{k}}{\left(b_{1}\right)_{k} \ldots\left(b_{q-1}\right)_{k}} \frac{z^{k}}{k !},
$$

where the factors $(\alpha)_{k}$ are called Pochhammer symbols and which are defined by:

$$
(\alpha)_{k}=\frac{\Gamma(\alpha+k)}{\Gamma(\alpha)} .
$$

For these functions the convergence conditions are:

- The generalized hypergeometric functions are given by series defined in the convergence region $|z|<1$, while for $|z|>1$ they are defined by analytical continuation.

- If $|z|<1$ the series converges absolutely. Since the variable $z$ represents the ratio between two energy scales of the topology, what is obtained is one of the limit representations or solutions, an expansion around $z \rightarrow 0$.

- If $z=1$, the necessary requirement for the series convergence is that $\Re e(\omega)>0$, where $\omega$ is called parametric excess and it is given by the equation:

$$
\omega=\sum_{j=0}^{q} b_{j}-\sum_{j=0}^{q+1} a_{j} .
$$

- For the convergence in $z=-1$ it is sufficient that $\Re e(\omega)>-1$.

\section{B.2 Some identities of the Pochhammer symbols}

The following identities are very useful for building the hypergeometric function starting from the contributions that are obtained of the MRE of an arbitrary diagram $G$. Aside from formula (139), sometimes it is useful to use other identities that are needed when there are factors of the type $\Gamma(a \pm n)$ and $\Gamma(a \pm 2 n)$ in the solutions, such as:

$$
\begin{gathered}
(a)_{-n}=\frac{\Gamma(a-n)}{\Gamma(a)}=\frac{(-1)^{n}}{(1-a)_{n}} \\
(a)_{2 n}=\frac{\Gamma(a+2 n)}{\Gamma(a)}=4^{n}\left(\frac{a}{2}\right)_{n}\left(\frac{a}{2}+\frac{1}{2}\right)_{n} .
\end{gathered}
$$

\section{B.3 Two variable hypergeometric functions}

Here we describe two variable hypergeometric functions, which correspond to the Kampé de Fériet generalized

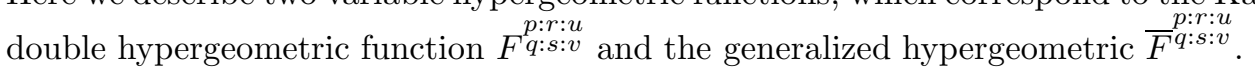




\section{B.3.1 Función Kampé de Fériet $\underset{F}{p: r: s: u}$}

This function is defined as:

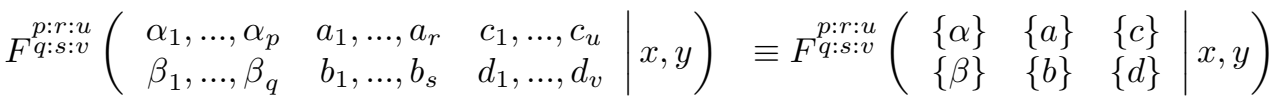

$$
\begin{aligned}
& \equiv \sum_{n, m}^{\infty} \frac{\prod_{j=1}^{p}\left(\alpha_{j}\right)_{n+m} \prod_{j=1}^{r}\left(a_{j}\right)_{n} \prod_{j=1}^{u}\left(c_{j}\right)_{m}}{\prod_{j=1}^{q}\left(\beta_{j}\right)_{n+m} \prod_{j=1}^{s}\left(b_{j}\right)_{n} \prod_{j=1}^{v}\left(d_{j}\right)_{m}} \frac{x^{n}}{n !} \frac{y^{m}}{m !},
\end{aligned}
$$

where the convergence conditions of the double series exist if the following relation between the indices are satisfied:

$$
\begin{aligned}
& p+r \leqslant q+s+1, \\
& p+u \leqslant q+v+1,
\end{aligned}
$$

and if furthermore, the arguments fulfil the condition:

$$
\begin{array}{ll}
|x|^{\frac{1}{(p-q)}}+|y|^{\frac{1}{(p-q)}}<1 & \text {, if }(p>q), \\
\max \{|x|,|y|\}<1 & \text {, if }(p \leqslant q) .
\end{array}
$$

\section{B.3.2 Function $\bar{F}^{p: r: s: v}$}

This series, which appears frequently in the solutions to Feynman diagrams, has the following definition:

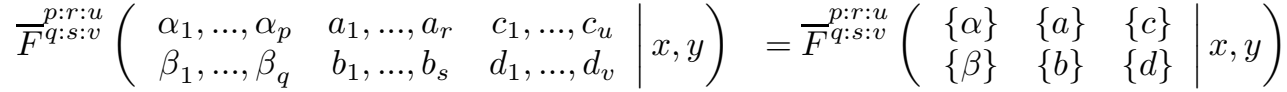

$$
\begin{aligned}
& =\sum_{n, m}^{\infty} \frac{\prod_{j=1}^{p}\left(\alpha_{j}\right)_{n-m} \prod_{j=1}^{r}\left(a_{j}\right)_{n} \prod_{j=1}^{u}\left(c_{j}\right)_{m}}{\prod_{j=1}^{q}\left(\beta_{j}\right)_{n-m} \prod_{j=1}^{s}\left(b_{j}\right)_{n} \prod_{j=1}^{v}\left(d_{j}\right)_{m}} \frac{x^{n}}{n !} \frac{y^{m}}{m !},
\end{aligned}
$$

where the convergence conditions of the double series exist if the following relation between the indices are satisfied:

$$
\begin{aligned}
& p+r \leqslant q+s+1, \\
& q+u \leqslant p+v+1 .
\end{aligned}
$$

All the series that have been found with the technique used here fulfil this indexes condition. The determination of the convergence region of the variables can be done using the Horns general convergence theory [21]. 


\section{Mathematical Formalism of the integration by fractional expan- sion Method}

\section{C.1 Introduction}

In section (2.2) we already introduced some algebraic aspects of the IBFE technique, which are generated from the identity associated to the integral parametrization of loops and which is known as Schwinger's parametrization:

$$
\frac{1}{A^{\beta}}=\frac{1}{\Gamma(\beta)} \int_{0}^{\infty} d x x^{\beta-1} \exp (-A x) .
$$

It is possible to find an operational equivalence between the integral symbol and a Kronecker delta, given by:

$$
\int d x x^{\beta+n-1} \equiv \Gamma(\beta) \frac{\Gamma(n+1)}{(-1)^{n}} \delta_{\beta+n, 0} .
$$

For simplicity we have eliminated the integral limits, since this identity has only validity in the context of the integrand expansion in (149). This expression is crucial for the development of the IBFE method, since in the Feynman diagram evaluation the corresponding Schwinger parametric representation is a generalized structure of the expression (149).

\section{C.1.1 Some Properties}

For the study of some properties of (150) it is convenient to use the notation defined in (13) in order to help us formalize the mechanism of the IBFE technique. Then let:

$$
\int d x x^{\nu_{1}+\nu_{2}-1} \equiv\left\langle\nu_{1}+\nu_{2}\right\rangle
$$

where $\nu_{1}$ and $\nu_{2}$ are indices which can take arbitrary values.

Property l. Commutativity of indices : We can explicitly write (151) in two possible forms according to formula (150):

$$
\left\langle\nu_{1}+\nu_{2}\right\rangle=\left\{\begin{array}{c}
\Gamma\left(\nu_{1}\right) \frac{\Gamma\left(\nu_{2}+1\right)}{(-1)^{\nu_{2}}} \delta_{\nu_{1}+\nu_{2}, 0}, \\
\Gamma\left(\nu_{2}\right) \frac{\Gamma\left(\nu_{1}+1\right)}{(-1)^{\nu_{1}}} \delta_{\nu_{1}+\nu_{2}, 0}
\end{array}\right.
$$

Starting from (149) one can show the equivalence of both forms in (152), and for this it is enough to expand the integrand exponential and replace $\langle\cdot\rangle$ for each case:

Let us consider the following integral representation:

$$
\frac{1}{A^{\nu_{1}}}=\frac{1}{\Gamma\left(\nu_{1}\right)} \int_{0}^{\infty} d x x^{\nu_{1}-1} \exp (-A x),
$$

which in terms of a series, in the proposed sense (151), turns out to be:

$$
\frac{1}{A^{\nu_{1}}}=\frac{1}{\Gamma\left(\nu_{1}\right)} \sum_{\nu_{2}} \frac{(-1)^{\nu_{2}}}{\Gamma\left(\nu_{2}+1\right)} A^{\nu_{2}}\left\langle\nu_{1}+\nu_{2}\right\rangle .
$$


Selecting now $\left\langle\nu_{1}+\nu_{2}\right\rangle=\Gamma\left(\nu_{1}\right) \frac{\Gamma\left(\nu_{2}+1\right)}{(-1)^{\nu_{2}}} \delta_{\nu_{1}+\nu_{2}, 0}$, we directly obtain the equality in (153). Analogously selecting $\left\langle\nu_{1}+\nu_{2}\right\rangle=\Gamma\left(\nu_{2}\right) \frac{\Gamma\left(\nu_{1}+1\right)}{(-1)^{\nu_{1}}} \delta_{\nu_{1}+\nu_{2}, 0}$ and using then the identity:

$$
\frac{\Gamma(y)}{\Gamma(y-z)}=(-1)^{-z} \frac{\Gamma(1+z-y)}{\Gamma(1-y)}
$$

with $y=\nu_{1}$ and $z=2 \nu_{1}$, the equality (153) is finally obtained, which shows the equivalence between the two ways of writing $\left\langle\nu_{1}+\nu_{2}\right\rangle$.

Another way of writing (151), which is useful for the simplification of terms that contain the factor:

$$
\frac{(-1)^{m}}{\Gamma(m+1)}
$$

where $m$ is an arbitrary index, is the following:

$$
\left\langle\nu_{1}+\nu_{2}\right\rangle=\left\langle\nu_{1}+\nu_{2}-m+m\right\rangle=\langle-m+m\rangle=\Gamma(-m) \frac{\Gamma(m+1)}{(-1)^{m}} \delta_{\nu_{1}+\nu_{2}, 0} .
$$

Notice that explicit use of the Kronecker deltas has been made, in order to simplify the parenthesis $\langle\cdot\rangle$, and since this happens in the context of expansions, the Kronecker deltas remain to indicate the constraints between the indices $\nu_{1}$ and $\nu_{2}$.

Property 11. Significance of the Multiregion Expansion MRE : Let us consider the following binomial expansion:

$$
\left(A_{1}+A_{2}\right)^{ \pm \nu}
$$

where the quantities $A_{1}, A_{2}$ and $\nu$ can take arbitrary values. In this case there are two possible regions or limits for the expansion: the region where $\left(A_{1}>A_{2}\right)$ and the region where $\left(A_{1}<A_{2}\right)$. These expansions are respectively:

1. Región $\left(A_{1}>A_{2}\right)$

$$
\left(A_{1}+A_{2}\right)^{ \pm \nu}=A_{1}^{ \pm \nu} \sum_{n=0}^{\infty} \frac{(\mp \nu)_{n}}{\Gamma(n+1)}\left(-\frac{A_{2}}{A_{1}}\right)^{n}
$$

2. Región $\left(A_{1}<A_{2}\right)$

$$
\left(A_{1}+A_{2}\right)^{ \pm \nu}=A_{2}^{ \pm \nu} \sum_{n=0}^{\infty} \frac{(\mp \nu)_{n}}{\Gamma(n+1)}\left(-\frac{A_{1}}{A_{2}}\right)^{n} .
$$

The factor $(\nu)_{n}$ is the Pochhammer symbol and it is given by:

$$
(\nu)_{n}=\frac{\Gamma(\nu+n)}{\Gamma(\nu)} .
$$

We have obtained in this manner expansions in the two possible limits separately. It is possible, however, to express both results employing a single series which contains simultaneously both regions. In this sense we can say that this type of expansion corresponds to a multiregion series representation of the binomial. To show this, let us express the binomial (158) using the integral representation of the denominator indicated in (149). Then we get:

$$
\left(A_{1}+A_{2}\right)^{ \pm \nu}=\frac{1}{\Gamma(\mp \nu)} \int_{0}^{\infty} d x x^{\mp \nu-1} \exp \left(-x A_{1}\right) \exp \left(-x A_{2}\right)
$$


and the exponentials are expanded separately, obtaining:

$$
\left(A_{1}+A_{2}\right)^{ \pm \nu}=\frac{1}{\Gamma(\mp \nu)} \sum_{n_{1}} \sum_{n_{2}} \frac{(-1)^{n_{1}+n_{2}}}{\Gamma\left(n_{1}+1\right) \Gamma\left(n_{2}+1\right)} A_{1}^{n_{1}} A_{2}^{n_{2}} \int d x x^{\mp \nu+n_{1}+n_{2}-1} .
$$

Using the identity (151) we get the multiregion binomial expansion:

$$
\left(A_{1}+A_{2}\right)^{ \pm \nu}=\frac{1}{\Gamma(\mp \nu)} \sum_{n_{1}} \sum_{n_{2}} \frac{(-1)^{n_{1}+n_{2}}}{\Gamma\left(n_{1}+1\right) \Gamma\left(n_{2}+1\right)} A_{1}^{n_{1}} A_{2}^{n_{2}}\left\langle\mp \nu+n_{1}+n_{2}\right\rangle,
$$

where according to property $(152)$ we can express the parenthesis $\langle\cdot\rangle$ in three different ways. In any of them we will have the same Kronecker delta which eliminates one of the two sums. Let us see:

$$
\left\langle\mp \nu+n_{1}+n_{2}\right\rangle=\left\{\begin{array}{l}
\Gamma\left(\mp \nu+n_{1}\right) \frac{\Gamma\left(n_{2}+1\right)}{(-1)^{n_{2}}} \delta_{\mp \nu+n_{1}+n_{2}, 0} \\
\Gamma\left(\mp \nu+n_{2}\right) \frac{\Gamma\left(n_{1}+1\right)}{(-1)^{n_{1}}} \delta_{\mp \nu+n_{1}+n_{2}, 0} \\
\Gamma\left(n_{2}+n_{1}\right) \frac{\Gamma(\mp \nu+1)}{(-1)^{\mp \nu}} \delta_{\mp \nu+n_{1}+n_{2}, 0} .
\end{array}\right.
$$

On the other hand, the number of possible ways of summing (164) using the Kronecker delta can be found in general by evaluating the combinatorial $C_{\text {Deltas }}^{\text {Summations }}$, which in this case is $C_{1}^{2}=2$. Let us see what happens when we sum with respect to one particular index:

\section{Sum respect to $n_{2}$}

Let us use for this case the following equality:

$$
\left\langle\mp \nu+n_{1}+n_{2}\right\rangle=\Gamma\left(\mp \nu+n_{1}\right) \frac{\Gamma\left(n_{2}+1\right)}{(-1)^{n_{2}}} \delta_{\mp \nu+n_{1}+n_{2}, 0},
$$

and then replacing in (164) we obtain:

$$
\left(A_{1}+A_{2}\right)^{ \pm \nu}=\frac{1}{\Gamma(\mp \nu)} \sum_{n_{1}}(-1)^{n_{1}} \frac{\Gamma\left(\mp \nu+n_{1}\right)}{\Gamma\left(n_{1}+1\right)} A_{1}^{n_{1}} A_{2}^{ \pm \nu-n_{1}},
$$

or equivalently:

$$
\left(A_{1}+A_{2}\right)^{ \pm \nu}=A_{2}^{ \pm \nu} \sum_{n_{1}=0}^{\infty} \frac{(\mp \nu)_{n_{1}}}{\Gamma\left(n_{1}+1\right)}\left(-\frac{A_{1}}{A_{2}}\right)^{n_{1}}
$$

which gives the expansion associated to the region $\left(A_{1}<A_{2}\right)$, obtained previously in (160).

\section{Sum respect to $n_{1}$}

Analogously, we now use the identity:

$$
\left\langle\mp \nu+n_{1}+n_{2}\right\rangle=\Gamma\left(\mp \nu+n_{2}\right) \frac{\Gamma\left(n_{1}+1\right)}{(-1)^{n_{1}}} \delta_{\mp \nu+n_{1}+n_{2}, 0},
$$

and replacing in (164) gives:

$$
\left(A_{1}+A_{2}\right)^{ \pm \nu}=A_{1}^{ \pm \nu} \sum_{n_{2}=0}^{\infty} \frac{(\mp \nu)_{n_{2}}}{\Gamma\left(n_{2}+1\right)}\left(-\frac{A_{2}}{A_{1}}\right)^{n_{2}}
$$

expression that was found in (159), and valid in the region $\left(A_{1}>A_{2}\right)$. 
The fundamental idea that has been exposed in the previous demonstration is that using the definition (150) it is possible to make a binomial expansion which differs from the conventional on the sense that the expansion is now around zero and infinity simultaneously. This can be generalized for multinomials and obtain its MRE:

$$
\left(A_{1}+\ldots+A_{l}\right)^{ \pm \nu}=\sum_{n_{1}} \ldots \sum_{n_{l}} \phi_{n_{1}, . ., n_{l}} A_{1}^{n_{1}} \ldots A_{l}^{n_{l}} \frac{\left\langle\mp \nu+n_{1}+\ldots+n_{l}\right\rangle}{\Gamma(\mp \nu)}
$$

The number of different expressions that can be extracted starting from equation (171) is given by all the possible forms of evaluating some of the sums, using for this purpose the Kronecker delta generated by the same expansion, that is $C_{1}^{n_{l}}=n_{l}$ possible forms. Generalizing even more, any function expressed of a multiregion series employing $\sigma$ sums and $\delta$ Kronecker deltas, has at most:

$$
C_{\delta}^{\sigma}=\frac{\sigma !}{\delta !(\sigma-\delta) !}
$$

possible ways of being evaluated, and each of these expansions corresponds to a series of multiplicity $\mu=$ $(\sigma-\delta)$.

All the resulting series are series representations with respect to the ratios between the terms of the multinomial and all of them correspond to multivariable generalizations of the hypergeometric function. 


\section{References}

[1] A. Grozin, Lectures on QED and QCD, Practical Calculation and Renormalization of One- and MultiLoop Feynman Diagrams, World Scientific Publishing Co. Pte. Ltd., Singapore, 2007; and references therein.

[2] V.A.Smirnov, Evaluating Feynman Integrals, Springer, Berlin, Heidelberg, 2004; and references therein.

[3] I.G.Halliday, R.M.Ricotta, Phys. Lett. B 193 (1987) 241.

[4] I.Gonzalez, I.Schmidt, Nucl. Phys. B 769 (2007) 124-173.

[5] I.Gonzalez, I.Schmidt, Phys.Rev. D72 (2005) 106006.

[6] C.Anastasiou, E.W.N.Glover, C.Oleari, Nucl.Phys. B572 (2000) 307-360 (hep-ph/9907494).

[7] A.T.Suzuki, E.S.Santos, A.G.M.Schmidt, Eur.Phys.J. C26 (2002) 125-137 (hep-th/0205158).

[8] A.T.Suzuki, E.S.Santos, A.G.M.Schmidt, J.Phys. A36 (2003) 4465 (hep-ph/0210148).

[9] A.T.Suzuki, A.G.M.Schmidt, J.Phys. A31 (1998) 8023-8039.

[10] C.Anastasiou, E.W.N.Glover, C.Oleari,Nucl.Phys. B565 (2000) 445-467 (hep-ph/9907523).

[11] A.T.Suzuki, A.G.M.Schmidt, Can.J.Phys. 78 (2000) 769-777 (hep-th/9904195).

[12] A.T.Suzuki, A.G.M.Schmidt, JHEP 9709 (1997) 002 (hep-th/9709024).

[13] A.T.Suzuki, A.G.M.Schmidt, Eur.Phys.J. C5 (1998) 175-179 (hep-th/9709144).

[14] A.T.Suzuki, A.G.M.Schmidt, Phys.Rev. D58 (1998) 047701 (hep-th/9712108).

[15] A.T.Suzuki, A.G.M.Schmidt, J.Phys. A35 (2002) 151-164 (hep-th/0110047).

[16] V. Rivasseau, From Perturbative to Constructive Renormalization, Princeton University Press, 1991.

[17] W.N.Bailey, Generalized Hypergeometric Functions, Stechert-Hafner service agency, New York and London, 1964.

[18] G.Gasper, M.Rahman, Basic Hypergeometric series, Cambridge University Press, 1990.

[19] L.J.Slater, Generalized Hypergeometric Functions, Cambridge University Press, 1966.

[20] L.S.Gradshteyn, L.M.Ryzhik, Table of Integrals, Series, and Products (Academic Press, New York, 2000), 6th ed.

[21] H. Exton, Multiple Hypergeometric Functions and Applications (Ellis Horwood,Westergate, England, 1976). 